\title{
Towards a Standardized Approach of Cetacean Habitat: Past Achievements and Future Directions
}

\author{
Nardi Cribb $^{1 *}$, Cara Miller ${ }^{2}$, Laurent Seuront ${ }^{3}$ \\ ${ }^{1}$ School of Biological Sciences, Flinders University, Adelaide, Australia \\ ${ }^{2}$ School of Biological and Chemical Sciences, Faculty of Science, Technology and Environment, The University \\ of the South Pacific, Laucala Campus, Suva, Fiji Islands \\ ${ }^{3}$ CNRS, UMR 8187 LOG, Wimereux, France \\ Email: nardi.cribb@flinders.edu.au
}

Received 19 May 2015; accepted 26 July 2015; published 30 July 2015

Copyright (C) 2015 by authors and Scientific Research Publishing Inc.

This work is licensed under the Creative Commons Attribution International License (CC BY).

http://creativecommons.org/licenses/by/4.0/

cC) (i) Open Access

\section{Abstract}

The understanding of what habitat means for an organism as well as the underlying factors driving patterns of habitat use is still unknown for many species. Cetacean habitat has been described using a range of methodologies and variables measured over various temporal and spatial scales that are often author-dependent. However, in order to develop an objective and sound understanding of what habitat actually means for cetaceans, a standardized approach needs to be developed. Here, after briefly reviewing the fundamental differences between terrestrial and marine habitats, we highlight the difficulty in defining a marine habitat, with a special focus on marine mammals. We subsequently provide six recommendations by which future cetacean habitat studies might be approached. This recommended approach aims to amend the way in which we think about and undertake investigations into cetacean habitat. It is believed that through this broadened approach, future cetacean habitat studies will increase our understanding of underlying driving factors of cetacean habitat, rather than just describing distribution patterns. Finally, it is stressed how the proposed approach will be more directly applicable within management frameworks and of benefit to conservation initiatives.

\section{Keywords}

Cetacean, Dolphins, Whales, Habitat, Modelling, Management, Conservation

\footnotetext{
${ }^{*}$ Corresponding author.
}

How to cite this paper: Cribb, N., Miller, C. and Seuront, L. (2015) Towards a Standardized Approach of Cetacean Habitat: Past Achievements and Future Directions. Open Journal of Marine Science, 5, 335-357. 


\section{Introduction}

The study of habitat is essential for understanding the biological and ecological requirements of animals as well as the strategies they employ to fulfill their needs [1]. In addition, habitat information is a fundamental prerequisite for the implementation of both management and conservation strategies [2]. However, the definition of habitat is still a contentious one, and its use is far from being consistent [3]. In particular, there is a general lack of unified definition in both terrestrial and marine ecological studies (Table 1 and Table 2). As a further example, a review of the use of the term in terrestrial studies found that $82 \%$ of articles reviewed, used habitat terminology imprecisely [4].

Table 1. Non-exhaustive review of habitat definitions applied and/or discussed in the terrestrial ecology literature.

\begin{tabular}{|c|c|}
\hline Definition & Reference \\
\hline $\begin{array}{l}\text { A species or population unit; an abstraction of the essential physical factors } \\
\text { and theco-habitant biota, in a locality where individuals of that population regularly live and reproduce }\end{array}$ & [82] \\
\hline Place/space where an organism lives & [83] \\
\hline The area of land, water and airspace required for the normal needs and survival of a species & [84] \\
\hline Area in which a wildlife community exists & [85] \\
\hline Location in which organisms live, or characterized by predominant plant or animal life & [13] \\
\hline $\begin{array}{l}\text { Resources and conditions present in an area that produce occupancy—including survival } \\
\text { and reproduction—by a given organism }\end{array}$ & [4] \\
\hline Where an animal lives that can be characterized by dominant plant forms or physical features & [86] \\
\hline $\begin{array}{l}\text { The resources an conditions present in an area that produce occupancy, } \\
\text { including survival and reproduction, by a given organism }\end{array}$ & [3] \\
\hline $\begin{array}{l}\text { Place where an animal lives, or, the collection of resources and conditions necessary for its occupancy, or, } \\
\text { a set of specific environmental features that, is equated to a plant community, vegetative association or cover type }\end{array}$ & [87] \\
\hline A place where an animal resides & [88] \\
\hline The abiotic components of the environment only & [11] \\
\hline $\begin{array}{c}\text { The physical and chemical components of an organism's environment, including the biotic environment } \\
\text { to emphasize that an organism must integrate and adapt to all the elements of its surroundings } \\
\text { including those that are living and those that are not }\end{array}$ & [89] \\
\hline $\begin{array}{c}\text { Description of the physical space, at a particular scale of space and time, } \\
\text { where an organism actually or potentially lives }\end{array}$ & [10] \\
\hline
\end{tabular}

Table 2. Non-exhaustive review of habitat definitions applied to general or specific groups of marine organisms.

\begin{tabular}{|c|c|c|}
\hline Definition & Species/order/taxa & Reference \\
\hline $\begin{array}{c}\text { A place in which a fish, a population or assemblage can find the physical or chemical } \\
\text { features required for life, e.g. suitable water quality, migration routes, spawning grounds, } \\
\text { feeding sites, resting sites, and shelter from predators and adverse weather }\end{array}$ & Fish & {$[90]$} \\
\hline Areas vital to the survival of a marine species at some phase in its life cycle & Various species & {$[91]$} \\
\hline The functioning ecological units required for successful breeding and foraging & Mammals & {$[92]$} \\
\hline The place where an organism can be found & Various species & {$[93]$} \\
\hline $\begin{array}{c}\text { Each species lives within a certain environment, whereby it has a preference for } \\
\text { a combination of environmental factors, e.g. substratum, temperature, salinity } \\
\text { and hydrodynamic conditions that it is able to live within }\end{array}$ & Various species & {$[94]$} \\
\hline $\begin{array}{c}\text { Parts of a cetacean's range, either a species or population of that species, essential } \\
\text { for the day-to-day survival, as for maintaining a healthy population growth rate. } \\
\text { Areas used for feeding, breeding, raising calves, migrating }\end{array}$ & Cetaceans & {$[7]$} \\
\hline $\begin{array}{c}\text { Features related to basic needs e.g. prey; refuge from predators; suitable conditions } \\
\text { for reproduction including mating and rearing young, resting, and moulting; } \\
\text { and safety from extreme environmental events }\end{array}$ & Mammals & [89] \\
\hline
\end{tabular}


Definition and research into habitat have frequently been identified as crucial for cetacean management and conservation (e.g. [2] [5]-[8]). However, a consistent definition and understanding of what habitat actually means for cetaceans is still lacking. As a consequence, there is limited information and understanding of habitat characteristics for most species [9]. In order to advance our understanding of the underlying drivers and processes that influence cetacean habitat, studies need to develop more a standardized and objective approach in which to examine them. This broadened approach will ultimately assist in the development and implementation of effective management, conservation and threat mitigation strategies.

In this context, the aims of the present work are: 1) to provide what we believe are representative examples between terrestrial and marine environments; 2) to highlight the specific features of marine environments that may contribute to the current lack of consensus in defining cetacean habitat, 3) provide a non-exhaustive review of how cetacean habitat has previously been studied, including modeling approaches and 4) to provide objective recommendations on how to develop an approach to studying habitat in order to advance cetacean ecology, and ultimately conservation and management efforts.

\section{Terrestrial versus Marine Habitats}

Typically, habitat in its simplest terms is defined as the physical environment, where an organism actually or potentially lives [10]. In addition, it has also been expanded to include the resources and environmental features present in an area which influences occupancy [4]. Habitat can also be thought of as a concept, used to link potential relationships between an organism and its physical and chemical environment [11]. However, a mechanistic understanding of this concept and how particular features influence organisms is still critically lacking [10].

Accurately describing and understanding the processes that determine the distribution of organisms is often constrained by the environment itself. Terrestrial and marine ecosystems are both spatially heterogeneous, comprised of ecological entities such as forests, hills, deserts, sea grass beds, seamounts and coral reefs, but also vary in time from diel to annual cycles (e.g. [12]). In terrestrial ecosystems, habitat is often defined by the presence of relatively persistent vegetation and animal life [13]. For example, the boundaries between the biotic and abiotic properties characterizing structurally diverse terrestrial environments (Figure 1) are easily observed and identifiable (e.g. vegetation patches, sedimentary rocky areas, gorges and slopes of cobbles and boulders). In many cases, the relatively immediate accessibility and visibility of the terrestrial environment, enhances our capacity to identify and observe environmental differences.

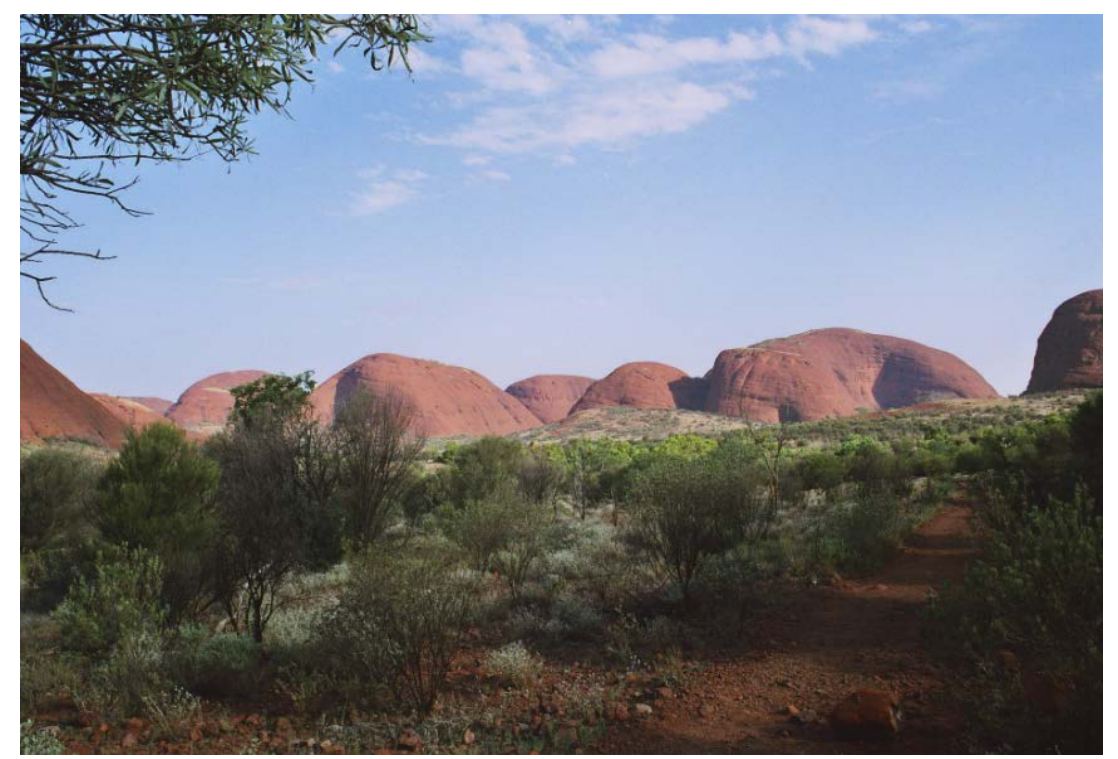

Figure 1. A terrestrial landscape, the Kata Tjuta (Northern Territory, Australia), illustrating how the boundaries between the biotic and abiotic features of a structurally diverse two-dimensional terrestrial habitat are easily identifiable and quantifiable, e.g. vegetation patches, sedimentary rocky areas, gorges and slopes of cobbles and boulders. Image credit: L. Seuront. 
In contrast, most marine environments are characterized by a limited number of landmarks both above and beneath the surface (Figure 2). Marine organisms typically live in a fluctuating and heterogeneous three-dimensional water mass. In addition, the inaccessibility of most of the World's ocean, and the logistical considerations inherent in effectively studying marine organisms once underwater, places additional limitations on how to define habitat for an organism, a species or a community. The characteristic wide-ranging and migratory nature of many marine animals, including cetaceans, often means that habitat boundaries are difficult to define ([7]; Figure 2), and may change on a temporal basis. Furthermore, regions within the World's Oceans are often defined by broad, general definitions such as open ocean or coastal waters, although specific sea surface temperature signatures such as warm and cold core eddies (Figure 3(A)) and thermal frontal zones (Figure 3(B)) can be specified. These broad classifications are frequently applied to species such as cetaceans, particularly those rarely sighted or cryptic species [14]. While these areas may be relatively distinct (Figure 3(C)), general classifications still lack a definitive understanding of what habitat actually means. As a consequence, the definition of marine habitat often seems arbitrary and in most cases non-existent. The application of the term habitat is often inconsistent even between marine animals of the same species or taxa (Table 2). These definitions highlight those potential factors (e.g. environmental factors) considered to be essential for the animals but again lack a thorough consideration of how the animal actually interacts with and relies on its environment.

In addition, habitat for many organisms (e.g. migratory birds, cryptic species), is often characterized using a limited number of observations recorded at specific encounter locations. Cetaceans are no exception, with habitat often described using only sightings or environmental measurements recorded at the surface, when the animals are exposed [15]-[19]. This strategy, however, disregards the properties and characteristics of the habitat concealed underneath, vertical structure of the water column. In contrast to terrestrial systems where environmental features are readily accessible and visible (Figure 1), in the marine environment it is considered much more difficult to gather relevant habitat information at depth. This again, potentially highlights the inaccessibility and the logistics of conducting research within these areas. Whilst some techniques (e.g. remote sensing imagery, animal borne sensors) can offer new perspectives and insight into detailed understanding of the vertical structure of the water column, they do not directly address the issue of habitat in order to provide sufficient information. Hence, in the marine environment, little information about the relations between species and their specific environments exists, despite their significance [20].

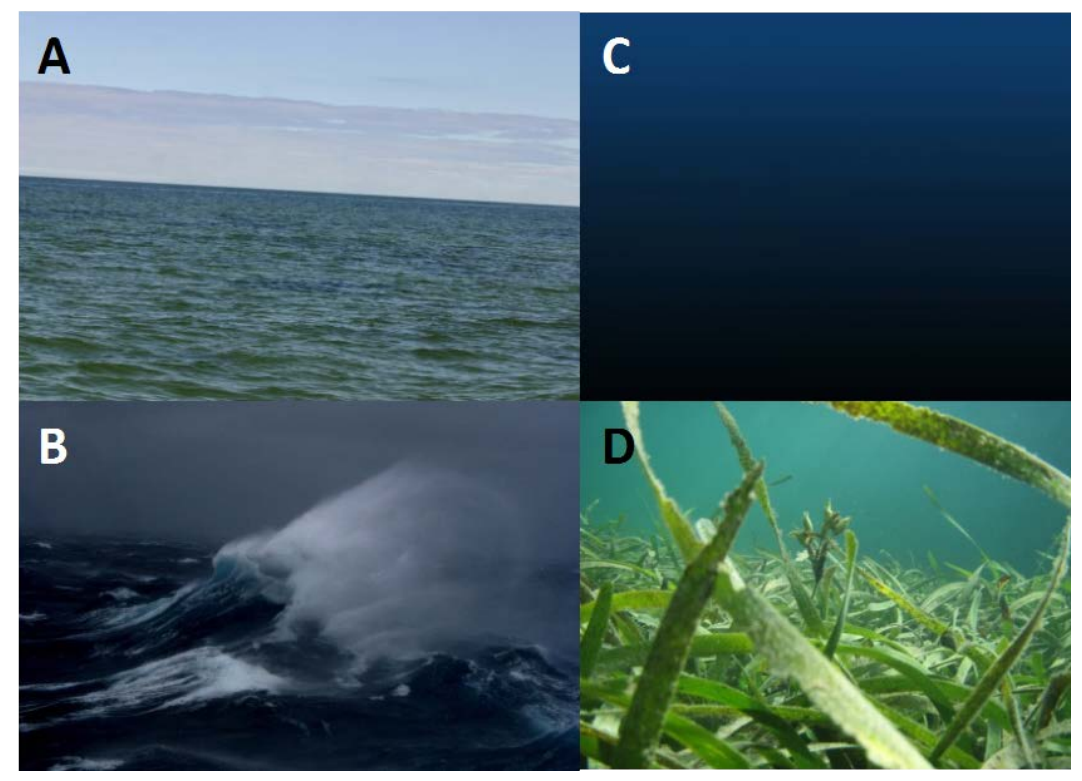

Figure 2. Marine landscapes, seen from above the surface in Gulf St. Vincent, South Australia (A) in stormy weather in the Southern Ocean ((B); $53^{\circ} \mathrm{S}, 145^{\circ} \mathrm{E}$ ), and beneath the surface in open water (C) above a seagrass bed in Louth Bay, South Australia (D), illustrating the difficulties in identifying landmarks and both abiotic and biotic properties leading to define cetacean habitat. Image credits: N. Cribb (A); V. Van Dongen-Vogels (B); L. Seuront ((C), (D)). 

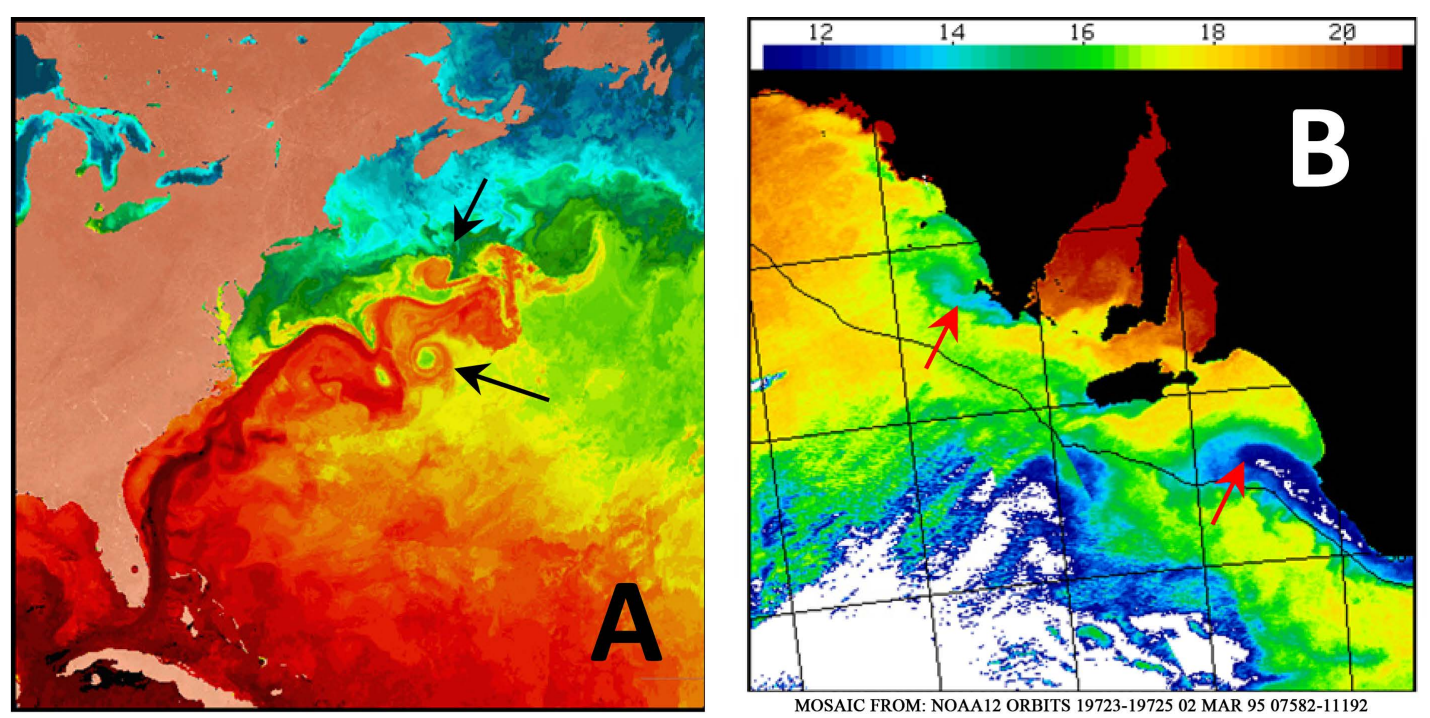

COPYRIGHT 1995, CSIRO MARINE LABORATORIES REMOTE SENSING FACILITY

SST (6 day composite): 16-Jul-2009-21-Jul-2009. Sealevel contours (0.1 m) and geostrophic velocity: 19-Jul-2009.

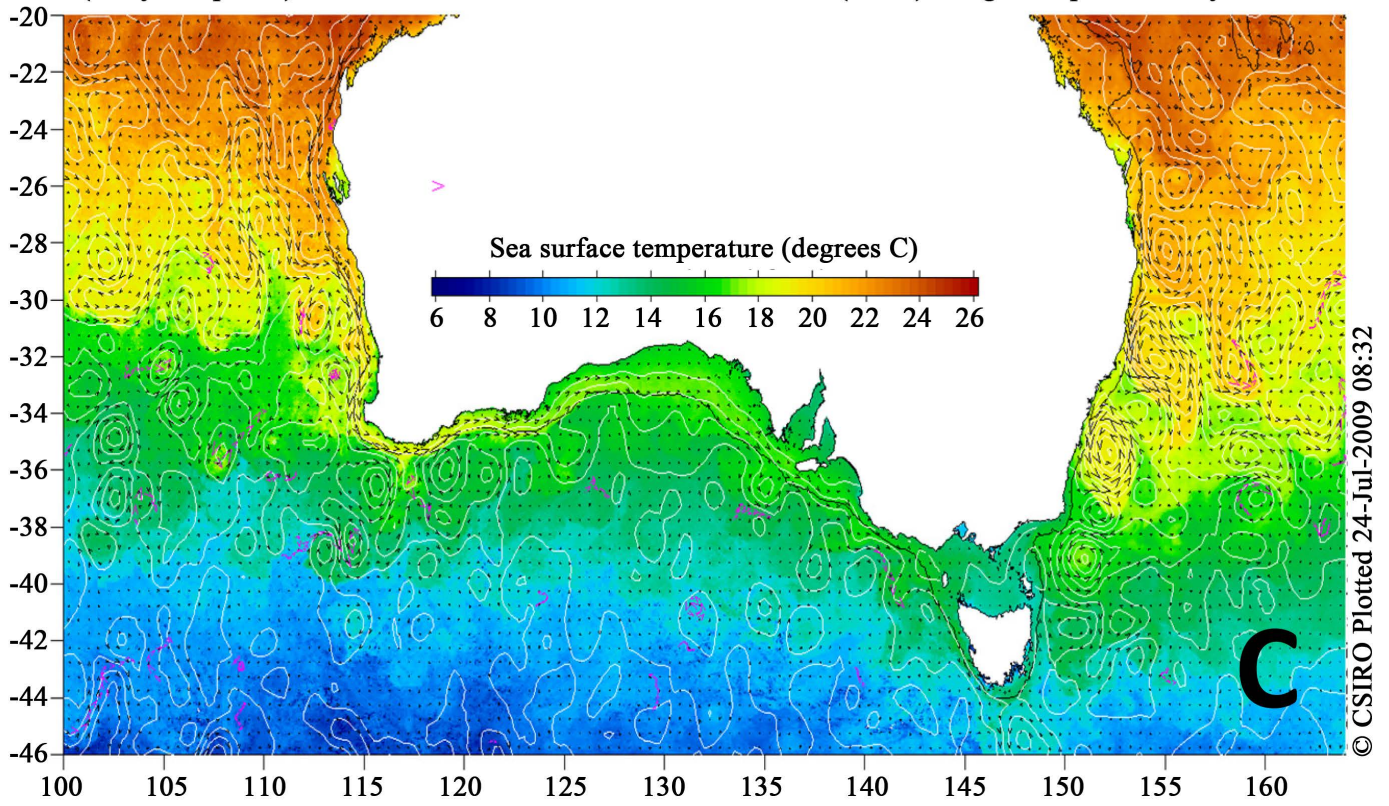

Figure 3. Illustration of typical sea surface temperature signatures of (A) the meandering Gulf Stream showing basin-scale thermal fronts and related warm and cold core eddies (black arrows); (B) upwelling events on South Australian shelf waters (red arrows), and (C) the Australian subtropical front that may be used to identify provinces inhabited by various cetacean species. Image credit: Ocean Remote Sensing Group, Johns Hopkins University Applied Physics Laboratory (A), and CSIRO ((B); (C)).

\section{The Concept of Cetacean Habitat So Far}

Cetacean species exhibit a wide range of distribution patterns across all parts of the World's Oceans [5] [21]-[26] As individuals and populations range widely and are not easy to observe directly, the concept of habitat is therefore difficult to grasp and define [27]. The wide diversity of cetacean species in general, makes our ability to understand their habitat more problematic. For example, many delphinids are widely distributed, with smaller populations inhabiting various locations and climatic regions, whilst in contrast larger mysticetes follow migrational routes each year to familiar calving areas [28]. The intrinsic difference in distributions across temperate and tropical, and coastal and offshore waters between the cetacean species, not only demonstrates their great ecological flexibility [29], but also ultimately links them to their habitat. In addition, the distinction between life 
history strategies and the biological requirements of the different species potentially influences their choice, and utilization of specific habitats in the marine environment; something which is also commonly dismissed in many habitat studies.

Critical cetacean habitat in a broad sense has previously been defined as "those parts of a cetacean's range either a species or population that are essential for the day-to-day survival and maintenance of a steady population growth rate, including those areas essential for specific behaviors such as mating, feeding and migrational routes" [7]. Cetacean habitat, and more specifically delphinid habitat, has typically been defined by investigating a number of abiotic and biotic factors ranging over various spatial and temporal scales thought to drive their distribution [30]-[36]. Factors previously investigated range from the physical and chemical features of the environment, such as water temperature, depth, salinity, topography and distance from shore, benthic habitat characteristics, and the presence of vessels, and preys and predators (Table 3). Measurement of these habitat variables was typically obtained using a wide variety of methodologies (e.g. in situ measurements, remotely sensed, obtained by boat or land based techniques), levels of precision (in situ vs. remotely sensed) and scales (temporal, spatial). Furthermore, variables used to assess habitat (even for a given species) were typically author and study dependent. For instance, a non-exhaustive review of pertinent studies of bottlenose dolphins (Tursiops sp.) clearly demonstrates the variety of both habitat measurements and spatio-temporal scales used [17] [37]-[40].

Among the variables used to assess cetacean habitat, sea surface temperature represents a common measurement that is often measured with a variety of scales and resolutions (Figure 3). For instance, [17] compared the distribution of white-sided (Lagenorhynchus acutus) and common (Delphinus delphis) dolphins off the coast of New Zealand with sea surface temperature and salinity measured from the survey vessel at the time of a dolphin sighting. In comparison, water temperature measurements accessed from remote sensing data was used to investigate seasonal distribution changes in striped dolphins (Stenella coeruleoalba) in the Ligurian Sea [41]. Of further note is the typically small number of environmental variables measured in many studies despite the high plausibility of other factors being instructive in describing habitat [42]-[44]. This consideration and narrowed selection of assessed variables should therefore caution our application of some habitat studies for progressing management objectives and conservation strategies. More specifically, a thorough understanding of constraints, methodology and objectives needs to be made to ensure that the results of habitat studies are not being confounded by the concentration of the researcher's effort and measurement tools or access [45].

\section{On the Contribution of Habitat Modeling to Cetacean Ecology}

Statistical habitat modeling, although still a relatively recent topic of research, is increasingly being applied to help answer questions regarding the ecology of many cetacean species [46]. Since the first paper there has been a significant growth and increase in this topic (Figure 4), which suggests a field in rapid development.

Typically, the aim of statistical habitat modeling is to help predict and explain variation in the distribution and density of cetaceans, as well as to predict key locations by correlating observations of animals with various environmental variables [47] [48]. However, these efforts may describe correlations between variables, but they generally lack the ability to elucidate our ecological understanding of the relationships between cetaceans and their marine environment.

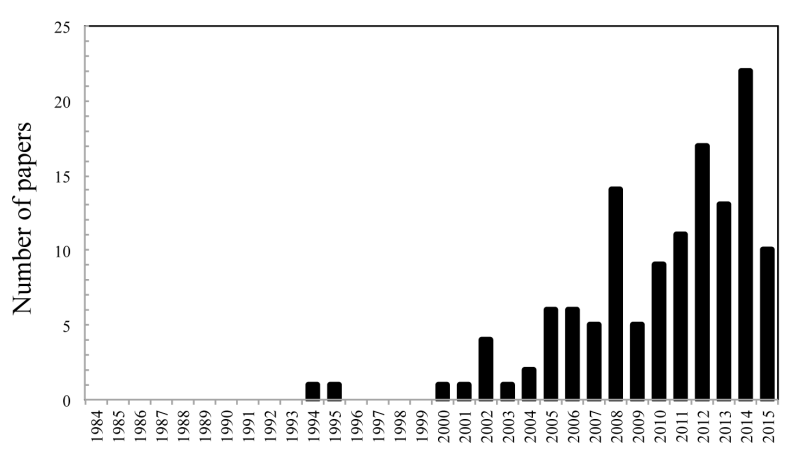

(A)

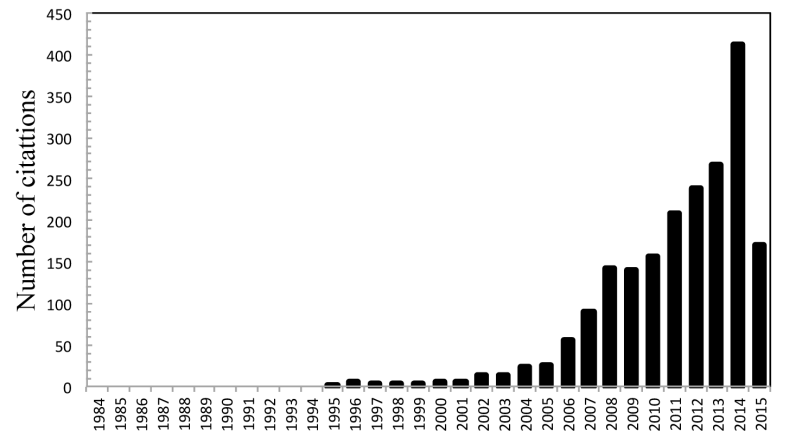

(B)

Figure 4. Number of papers containing the words cetacean, habitat and model in their topics published per year over the last 20 years (A) and their subsequent number of citations per year (B). 
Table 3. Common name, location and examples of variables used to define habitat and distribution in global delphinid studies from 1968 to present.

\begin{tabular}{|c|c|c|c|}
\hline Common name & Location & Variables used to define habitat & Reference \\
\hline $\begin{array}{l}\text { Common dolphin, Dusky dolphin, } \\
\text { Hourglass dolphin }\end{array}$ & South Pacific & $\mathrm{SST}^{\mathrm{a}}$ & [15] \\
\hline Common bottlenose dolphin & Gulf of Mexico & $\begin{array}{c}\text { Distribution patterns related to tidal occurrence, } \\
\text { time of day, season }\end{array}$ & {$[95]$} \\
\hline White-sided dolphin, Common dolphin & North Atlantic & SST, salinity, bottom topography & {$[95]$} \\
\hline Common bottlenose dolphin & Moreton Bay & Distance from shore & {$[96]$} \\
\hline Common bottlenose dolphin & Gulf of California & $\begin{array}{l}\text { Behavioral and range patterns related to estuarine } \\
\text { vs. non-estuarine habitat types, depth, Secchi depth }\end{array}$ & [97] \\
\hline Pilot whale & North Atlantic & Depth, bottom topography, SST & {$[29]$} \\
\hline Risso’s dolphin & Gulf of Mexico & Depth, depth gradient & {$[42]$} \\
\hline Common bottlenose dolphin & Moray Firth & $\begin{array}{l}\text { Movement patterns and seasonal distribution } \\
\text { determined through photo-identification }\end{array}$ & {$[16]$} \\
\hline Indo-Pacific humpback dolphin & Algoa Bay & $\begin{array}{l}\text { Distance to shore, depth, behavioral } \\
\text { activities related to physical habitat features }\end{array}$ & [98] \\
\hline Tursiops sp. & Shark Bay & Reproductive success, depth, SST & [99] \\
\hline Common bottlenose dolphin & Gulf of Mexico & $\begin{array}{l}\text { Foraging behavior, prey presence related } \\
\text { to benthic habitat characteristics }\end{array}$ & {$[100]$} \\
\hline Hector's dolphin & Porpoise Bay & $\begin{array}{l}\text { Photo-identification \& land based theodolite fixes to } \\
\text { determine spatial, temporal distribution patterns }\end{array}$ & {$[101]$} \\
\hline Indo-Pacific bottlenose dolphin & Shark Bay & $\begin{array}{l}\text { Effect of prey abundance, shark presence } \\
\text { on habitat use \& group size }\end{array}$ & {$[31]$} \\
\hline Tursiops sp. & Shannon estuary & $\begin{array}{l}\text { Encounter locations related to benthic } \\
\text { topography, depth, benthic slope }\end{array}$ & {$[38]$} \\
\hline Common bottlenose dolphin & Moray Firth & $\begin{array}{l}\text { Spatial, temporal distribution related } \\
\text { to tidal cycle, tidal front }\end{array}$ & {$[102]$} \\
\hline $\begin{array}{l}\text { Common bottlenose dolphin, } \\
\text { Atlantic spotted dolphin }\end{array}$ & Gulf of Mexico & Depth, SST, salinity, Chl. $a^{\mathrm{b}}$ & {$[103]$} \\
\hline Common bottlenose dolphin & Moray Firth & $\begin{array}{l}\text { Foraging observations related to local } \\
\text { submarine habitat characteristics }\end{array}$ & {$[32]$} \\
\hline Common bottlenose dolphin & Chesapeake Bay & $\begin{array}{l}\text { SST and Chl. } a \text { used as surrogates to } \\
\text { monitor dolphin and prey movements }\end{array}$ & {$[104]$} \\
\hline Common bottlenose dolphin & Mid-Atlantic Bight & SST, Chl. $a$ & {$[105]$} \\
\hline $\begin{array}{l}\text { Atlantic spotted dolphin, Pantropical } \\
\text { spotted dolphin, Clymene dolphin, } \\
\text { Spinner dolphin, Striped dolphin }\end{array}$ & South West Atlantic & Depth, SST & {$[106]$} \\
\hline $\begin{array}{l}\text { Snubfin dolphin, } \\
\text { Indo-Pacific humpback dolphin }\end{array}$ & Cleveland Bay & $\begin{array}{l}\text { SST, proximity to environmental } \\
\text { features, bathymetry }\end{array}$ & {$[107]$} \\
\hline Peale’s dolphin & Straight of Magellan & $\begin{array}{l}\text { Dolphin presence and behavioral } \\
\text { activities related to kelp beds }\end{array}$ & {$[108]$} \\
\hline Tursiops sp. & Shark Bay & Aquaculture presence & {$[33]$} \\
\hline Tursiops sp. & Shark Bay & Vessel effect on abundance & {$[34]$} \\
\hline Spinner dolphin & $\begin{array}{l}\text { Central } \\
\text { Tropical Pacific }\end{array}$ & $\begin{array}{l}\text { Surface turbidity, current, swell height, } \\
\text { distance to shore, vessel presence }\end{array}$ & {$[34]$} \\
\hline $\begin{array}{l}\text { Snubfin dolphin, Indo-Pacific } \\
\text { humpback dolphin }\end{array}$ & Cleveland Bay & $\begin{array}{l}\text { Depth, distance to physical } \\
\text { environmental features }\end{array}$ & [109] \\
\hline $\begin{array}{l}\text { Common dolphin, Striped dolphin, } \\
\text { Common bottlenose dolphin, } \\
\text { Harbour porpoise, Pilot whale }\end{array}$ & English Channel & $\begin{array}{l}\text { Distribution, encounter rate, } \\
\text { bathymetric preference }\end{array}$ & [19] \\
\hline
\end{tabular}


Continued

\begin{tabular}{|c|c|c|c|}
\hline Striped dolphin & Ligurian Sea & Chl. $a$, SST & [41] \\
\hline Common dolphin & Mediterranean Sea & $\begin{array}{l}\text { Calf presence, inter-specific relationships, } \\
\text { behavior, Chl. } a \text {, SST, depth, slope of seabed }\end{array}$ & [110] \\
\hline Indo-Pacific bottlenose dolphin & Gulf St Vincent & $\begin{array}{l}\text { Depth, SST, salinity, dissolved oxygen, turbidity, } \\
\text { distribution in relation to benthic characteristics }\end{array}$ & [39] \\
\hline Harbour porpoise & English Channel & Sightings related to diurnal and tidal patterns & [111] \\
\hline Common bottlenose dolphin & $\begin{array}{l}\text { Barataria and } \\
\text { Caminada Bays }\end{array}$ & $\begin{array}{l}\text { SST, salinity, dissolved oxygen, depth, } \\
\text { turbidity, distance to shore }\end{array}$ & [40] \\
\hline Spinner dolphin & Red Sea & SST, distribution related to swimmer presence & [57] \\
\hline Common dolphin & Gulf St Vincent & Depth, SST, latitude, longitude & [112] \\
\hline
\end{tabular}

${ }^{\mathrm{a}} \mathrm{SST}$ : Sea Surface Temperature; ${ }^{\mathrm{b}} \mathrm{Chl}$. $a$ : chlorophyll $a$ concentration.

From a non-exhaustive review, we show as aforementioned for field-based habitat studies that modeling habitat studies do not converge in their approaches, methodologies, spatial and temporal scales and analyses even when they target the same species (Table 4). Some studies are vague in their definition of a potential focus species as well as an ecological question, and often the focus species is then defined afterwards depending upon what species were observed during surveys. The overall objective of many studies is then often limited to predict where and when cetaceans are present (e.g. [49]). However, some studies do attempt to explain this presence further by linking them to features of the physical and biological oceanographic properties of their environment [48]; these properties have either been assessed using remote sensing data (e.g. sea surface temperature, sea surface height), variables measured in situ (e.g. depth, mixing layer thickness) or even modeled environmental data such as prey densities [50], hence allow to cover a very wide range of spatial and temporal scales; see Table 4 for further examples. There is, however, a strong study-to-study variability in the abiotic properties considered even in modeling studies dealing with similar environments and species (Table 4). Biotic variables are also dramatically under-represented (especially when compared to physical variables) in most of the studies reported here (Table 4). Similarly, biotic factors that may be critical to understand cetacean habitat use such as behavioral and life history strategies, have still been seldom used in habitat modeling studies ([48]; Table 4). Besides, studies that incorporate field-based visual and acoustic surveys [51]-[53] often lack information about the physical and vertical properties of the environments (Table 4). Also note that most synoptic studies that used remote sensing data, critically lack information about the vertical structure of the water column [54]. The aforementioned limitations of habitat modeling studies_-which are by no means a criticism of their results and do not detract from the central point of their work-hence suggest that although habitat modeling studies provide valuable information on where and when cetaceans may be over space and time, they still ultimately lack the power in which to truly understand the mechanistic links between the presence and behavior of cetaceans and the nature of their environment.

As a conclusion, statistical habitat modeling is undeniably a useful and promising tool to predict cetacean distributions as a function of range of descriptors (Table 4), in particular for those large whale and offshore cryptic species which lack baseline data and are often difficult to access. However, this approach still does not converge in the approach followed (Table 4), hence may prevent future progress in our ability to provide further insight into animal ecology. As stressed in the present work and in the recommendation below, there is a genuine need to refine modeling methods to move beyond correlations towards a mechanistic understanding of the processes that interact to create cetacean habitat and try to provide a more ecological explanation for their presence. Ultimately, this may also help to bridge the gap between fundamental research and conservation and management initiatives.

\section{How to Fill in the Gaps?}

To increase our understanding of cetacean habitat, we suggest to develop a more systematic and objective approach to cetacean habitat research. In particular, we stress the need to identify the underlying influences driving habitat, for example physical and chemical environmental features, social and behavioral factors, predation and anthropogenic pressures in order to determine how cetaceans interact with and use their environment. With this 
Table 4. Location, species and environmental variables used in habitat modeling studies to define cetaceans and habitat distributions.

\begin{tabular}{|c|c|c|c|c|}
\hline \multirow{2}{*}{ Location } & \multirow{2}{*}{ Species } & \multicolumn{2}{|c|}{ Environmental variables used to model habitat } & \multirow{2}{*}{ Reference } \\
\hline & & Abiotic & Biotic & \\
\hline Nova Scotia & $\begin{array}{c}\mathrm{M}_{3}, \mathrm{M}_{4}, \mathrm{M}_{7}, \mathrm{M}_{8}, \mathrm{O}_{1}, \mathrm{O}_{5} \\
\mathrm{O}_{8}, \mathrm{O}_{15}, \mathrm{O}_{19}, \mathrm{O}_{24}, \mathrm{O}_{25}\end{array}$ & Depth, slope, $\mathrm{SST}_{\text {in situ }}{ }^{\mathrm{a}}$ & & {$[113]$} \\
\hline California & $\mathrm{O}_{25}, \mathrm{O}_{31}$ & $\mathrm{SST}_{\text {in situ, }}$ salinity, depth & & {$[114]$} \\
\hline British Columbia & $\mathrm{M}_{3}, \mathrm{M}_{4}, \mathrm{M}_{5}, \mathrm{M}_{8}, \mathrm{O}_{1}$ & Depth, slope, $\mathrm{SST}_{\text {in situ }}$, salinity, & $\begin{array}{c}\text { Historical } \\
\text { whaling data }\end{array}$ & {$[115]$} \\
\hline $\begin{array}{l}\text { Mid-west North } \\
\text { Atlantic Ocean }\end{array}$ & $\begin{array}{c}\mathrm{M}_{4}, \mathrm{M}_{7}, \mathrm{M}_{8}, \mathrm{O}_{1}, \mathrm{O}_{4}, \mathrm{O}_{8}, \mathrm{O}_{9}, \mathrm{O}_{15}, \mathrm{O}_{18} \\
\mathrm{O}_{19}, \mathrm{O}_{20}, \mathrm{O}_{21}, \mathrm{O}_{25}, \mathrm{O}_{32}, \text { Mesoplodon spp. }\end{array}$ & $\begin{array}{l}\mathrm{SST}_{\text {in situ }} \text {, front } \\
\text { occurrence, depth, slope }\end{array}$ & & {$[116]$} \\
\hline Spain & $\mathrm{O}_{1}, \mathrm{O}_{4}, \mathrm{O}_{5}, \mathrm{O}_{8}, \mathrm{O}_{18}, \mathrm{O}_{19}, \mathrm{O}_{24}, \mathrm{O}_{25}$ & Depth, slope, $\mathrm{SST}_{\text {sat }}$ & & {$[117]$} \\
\hline $\begin{array}{l}\text { Faroe-Shetland } \\
\text { Channel }\end{array}$ & Oceanic dolphins & $\begin{array}{l}\mathrm{SST}_{\text {in situ, }} \text {, salinity, depth, } \\
\text { slope, ambient noise }\end{array}$ & Chl. $a_{\text {in situ }}{ }^{\mathrm{b}}$ & {$[118]$} \\
\hline $\begin{array}{l}\text { Eastern } \\
\text { Tropical Pacific }\end{array}$ & $\mathrm{O}_{4}$, Mesoplodon spp. & $\begin{array}{l}\text { Thermocline depth and strength, } \\
\text { SST }_{\text {in situ }} \text {, salinity, depth, slope }\end{array}$ & Chl. $a_{\text {in situ }}$ & {$[50]$} \\
\hline $\begin{array}{l}\text { Eastern } \\
\text { Tropical Pacific }\end{array}$ & $\begin{array}{c}\mathrm{O}_{7}, \mathrm{O}_{8}, \mathrm{O}_{9}, \mathrm{O}_{10}, \mathrm{O}_{11}, \mathrm{O}_{12}, \mathrm{O}_{13}, \mathrm{O}_{14}, \mathrm{O}_{18} \\
\mathrm{O}_{19}, \mathrm{O}_{20}, \mathrm{O}_{22}, \mathrm{O}_{23}, \mathrm{O}_{24}, \mathrm{O}_{25}, \mathrm{O}_{26}, \mathrm{O}_{27}, \mathrm{O}_{28}\end{array}$ & $\begin{array}{l}\text { SST }_{\text {in situ }} \text {, salinity, thermocline } \\
\text { depth and strength, depth, slope }\end{array}$ & Chl. $a_{\text {in situ }}$ & {$[119]$} \\
\hline $\begin{array}{l}\text { Western } \\
\text { Antarctic Peninsula }\end{array}$ & $\mathrm{M}_{8}, \mathrm{M}_{6}$ & $\begin{array}{l}\text { Acoustic volume backscatter, } \\
\text { depth, slope, temperature }{ }_{\text {in situ }}\end{array}$ & Chl. $a_{\text {in situ }}$ & {$[120]$} \\
\hline South central Alaska & $\mathrm{O}_{2}$ & Depth, flow accumulation & & {$[121]$} \\
\hline Hawaiian Archipelago & $\mathrm{M}_{8}$ & Depth, $\mathrm{SST}_{\text {sat }}$ & & {$[51]$} \\
\hline $\begin{array}{l}\text { Northern } \\
\text { Adriatic Seas }\end{array}$ & $\mathrm{O}_{19}$ & $\begin{array}{c}\mathrm{O}_{2} \text { saturation, temperature } \\
\text { densitity anomaly, turbidity, } \\
\text { depth, salinity, } \mathrm{pH} \text {, turbidity }\end{array}$ & $\mathrm{IVF}^{\mathrm{c}}$ & {$[8]$} \\
\hline Bay of Biscay & $\mathrm{O}_{19}, \mathrm{O}_{24}, \mathrm{O}_{25}$ & Distance & & {$[122]$} \\
\hline SW Mediterranean Sea & $\mathrm{O}_{25}$ & $\begin{array}{l}\text { Depth, slope, } \text { SST }_{\text {sat }} \text {, } \\
\text { scattering layers }\end{array}$ & $\begin{array}{l}\text { Chl. } a_{\text {sat }} \text {, } \\
\text { presence of } \\
\text { calves, } \\
\text { interspecific } \\
\text { relationships, } \\
\text { behavior }\end{array}$ & {$[110]$} \\
\hline Strait of Gibraltar & $\mathrm{O}_{1}, \mathrm{O}_{7}, \mathrm{O}_{8}, \mathrm{O}_{19}, \mathrm{O}_{24}, \mathrm{O}_{25}$ & Depth, slope & & {$[123]$} \\
\hline $\begin{array}{l}\text { Central Mediterranean } \\
\text { Sea }\end{array}$ & $\mathrm{O}_{18}, \mathrm{O}_{19}, \mathrm{O}_{24}$ & Depth, slope, $\mathrm{SST}_{\text {sat }}$ & Chl. $a_{\mathrm{sat}}$ & {$[124]$} \\
\hline $\begin{array}{l}\text { Pelagos sanctuary } \\
\text { (Mediterranean Sea) }\end{array}$ & $\mathrm{M}_{4}, \mathrm{O}_{24}$ & Depth, slope, $\mathrm{SST}_{\mathrm{sat}}$ & Chl. $a_{\mathrm{sat}}$ & {$[125]$} \\
\hline $\begin{array}{l}\text { North-western } \\
\text { Mediterranean }\end{array}$ & $\mathrm{O}_{1}, \mathrm{O}_{9}, \mathrm{O}_{18}$ & $\begin{array}{l}\text { Depth, slope, } \mathrm{SST}_{\text {sat }} \text {, } \\
\text { fronts, salinity }\end{array}$ & Chl. $a_{\mathrm{sat}}$ & {$[52]$} \\
\hline Eastern tropical pacific & $\mathrm{O}_{24}, \mathrm{O}_{23}, \mathrm{O}_{25}, \mathrm{O}_{18}$ & $\begin{array}{l}\mathrm{SST}_{\text {in situ }} \text {, salinity, thermocline } \\
\text { depth and strength, } \\
\text { depth, temperature fronts }\end{array}$ & Chl. $a_{\text {in situ }}$ & {$[126]$} \\
\hline Mid Atlantic Ridge & $\mathrm{M}_{5}, \mathrm{O}_{1}$ & $\begin{array}{l}\text { Bathymetry, slope, flow velocity, } \\
\text { water level gradient, } \\
\text { temperature and salinity gradients }\end{array}$ & & {$[127]$} \\
\hline Florida Bay, USA & $\mathrm{O}_{19}$ & $\begin{array}{l}\text { Temperature }_{\text {in situ }} \text {, salinity, } \\
\text { turbidity, dissolved } \mathrm{O}_{2} \text {, } \\
\text { benthic type }\end{array}$ & $\begin{array}{l}\text { Chl. } a_{\text {in situ }} \\
\text { Dolphin prey } \\
\text { per unit effort }\end{array}$ & {$[128]$} \\
\hline Scotland & $\mathrm{O}_{19}, \mathrm{O}_{32}$ & $\begin{array}{l}\text { Depth, slope, distance, } \mathrm{SST}_{\text {in situ }} \text {, } \\
\text { sediment type, salinity }\end{array}$ & & {$[129]$} \\
\hline $\begin{array}{l}\text { Greater Minch, } \\
\text { Scotland }\end{array}$ & $\mathrm{O}_{32}$ & $\begin{array}{l}\text { Depth, bathymetry, distance, } \\
\text { tidal conditions }\end{array}$ & & {$[130]$} \\
\hline Gulf of Maine & $\mathbf{M}_{1}$ & & $\begin{array}{l}\text { Modeled prey } \\
\text { densities, arrival } \\
\text { date of whales in } \\
\text { study location, } \\
\text { sightings per } \\
\text { unit effort }\end{array}$ & {$[50]$} \\
\hline
\end{tabular}




\section{Continued}

$\begin{array}{cc}\begin{array}{c}\text { Sundarbans mangrove } \\ \text { forest, Bangladesh }\end{array} & \mathrm{O}_{6}, \mathrm{O}_{33} \\ & \mathrm{M}_{3}, \mathrm{M}_{4}, \mathrm{M}_{8}, \mathrm{O}_{1}, \mathrm{O}_{14}, \\ \text { California current } & \mathrm{O}_{18}, \mathrm{O}_{24}, \mathrm{O}_{25}, \mathrm{O}_{28}, \mathrm{O}_{31}\end{array}$

Western Mediterranean

$\mathrm{O}_{24}$

Inner Hebrides,

Scotland

Argentina

Chile

Oman

Patagonia, Argentina

Balearic Islands

Southern Californian Bight

Southern ocean
Hebrides, Scotland
Pelagos sanctuary
(Mediterranean Sea)

California current

Atlantic east coast and Gulf of Mexico

British Columbia

St Lawrence

River estuary, Canada

California current,

Eastern Tropical Pacific

Hawaiian Islands

Scotland, west coast

Scotland

Australasia
$\mathrm{O}_{32}$

$\mathrm{O}_{18}$

$\mathrm{O}_{32}$

$\mathrm{O}_{15}$

$\mathrm{M}_{3}, \mathrm{M}_{7}, \mathrm{M}_{8}, \mathrm{O}_{4}, \mathrm{O}_{7}, \mathrm{O}_{10}, \mathrm{O}_{17}, \mathrm{O}_{19}, \mathrm{O}_{30}$ unidentified mysticetes and unidentified odontocetes $\mathrm{M}_{8}$, Balaenoptera spp. $\mathrm{O}_{15}, \mathrm{O}_{29}$ $\mathrm{O}_{1}$

$$
\mathrm{O}_{14}, \mathrm{O}_{18}
$$

$\mathrm{M}_{7}$

$\mathrm{M}_{6}$

$$
\mathrm{M}_{4}, \mathrm{O}_{1}, \mathrm{O}_{4}, \mathrm{O}_{8}, \mathrm{O}_{18}, \mathrm{O}_{19}, \mathrm{O}_{24}
$$

$$
\mathrm{M}_{4}, \mathrm{O}_{24}, \mathrm{O}_{31}
$$

$\mathrm{M}_{1}, \mathrm{M}_{8}, \mathrm{O}_{1}, \mathrm{O}_{7}, \mathrm{O}_{19}, \mathrm{O}_{20}, \mathrm{O}_{21}, \mathrm{O}_{22}$, $\mathrm{O}_{24}, \mathrm{O}_{25}, \mathrm{O}_{32}$, Baleen whale spp.,

Beaked whale spp., Kogia spp., Lagenorhyncus spp., Pilot whale spp.,

$\mathrm{M}_{3}, \mathrm{M}_{4}, \mathrm{M}_{8}, \mathrm{O}_{1}, \mathrm{O}_{3}, \mathrm{O}_{4}, \mathrm{O}_{14}, \mathrm{O}_{18}, \mathrm{O}_{24}$, $\mathrm{O}_{25}, \mathrm{O}_{28}, \mathrm{O}_{31}$, Mesoplodon spp.

$\mathrm{O}_{22}$

$\mathrm{M}_{2}$
Salinity, depth, turbidity,

temperature $_{\text {in situ }}$, channel

width, convergences

$\mathrm{SST}_{\text {in situ }}$ sat, frontal regions,

oceanic zone, depth, slope

Absolute dynamic topography,

$\mathrm{SST}_{\text {sat, in situ }}$, absolute

geostrophic velocity, sea level

anomaly, geostrophic velocity anomaly, depth

Tidal conditions, depth,

slope, sediment type

Depth, slope, SST $_{\text {sat }}$

Depth, channel width, coastline complexity

Slope, depth

Depth, $\mathrm{SST}_{\mathrm{sat}}$

Chl. $a_{\mathrm{sat}}$

Depth, slope, SST $_{\text {sat }}$, SS height deviation, surface wind direction

Chl. $a_{\text {sat }}$

Echolocation click occurrence,

lunar duration, upwelling

index, $\mathrm{SST}_{\text {sat }}$, interaction

of week and region

Depth, sea ice cover,

distance, distance, slope

Bathymetry, slope, $\mathrm{SST}_{\text {sat }}$, tidal current, depth

Depth, slope

Chl. $a_{\text {sat }}$

Chl. $a_{\text {sat }}$

Chl. $a_{\text {sat }}$, sandeel occurrence

Depth, slope, $\mathrm{SST}_{\text {sat }}$

Depth, distance to shore, monthly SST distance to continental shelf break

Depth, slope, $\mathrm{SST}_{\text {sat }}$

Chl. $a_{\text {sat }}$

Depth, slope, feeding

Behavior

Depth, slope, $\mathrm{SST}_{\text {sat }}$, salinity, mixed layer depth

Chl. $a_{\text {in situ }}$

Aspect variety, bay area,

coastline to area of a bay ratio, depth, distance, proportion of bay area with depths $<250 \mathrm{~m}$

Depth, slope, current speed, tidal conditions, sediment type

Depth, slope, sediment type

Prey distribution

Depth, slope, temperature ${ }_{\text {sat }}$, mixed layer depth, currents
Historical

whaling data,

Chl. $a_{\text {sat }}$

${ }^{\mathrm{a} S e a}$ surface temperature referred to as $\mathrm{SST}_{\text {sat }}$ hereafter from satellite data, and as $\mathrm{SST}_{\text {in situ }}$ when measured in situ. ${ }^{\mathrm{b}} \mathrm{Chlorophyll} a$ concentration referred to as chla ${ }_{\text {sat }}$ from satellite data, as chla ${ }_{\text {in situ }}$ when measured in situ. ${ }^{\mathrm{C}} \mathrm{IVF}$ in vivo fluorescence measured in situ as a proxy of chlorophyll $a$ concentration. The letters $\mathrm{M}$ and $\mathrm{O}$ respectively stands for Mysticetes and Odontocetes; $\mathrm{M}_{1}$ : Eubalaena glacialis, $\mathrm{M}_{2}$ : Eubalaena australis, $\mathrm{M}_{3}$ : Balaenoptera musculus, $\mathrm{M}_{4}$ : Balaenoptera physalus, $\mathrm{M}_{5}$ : Balaenoptera borealis, $\mathrm{M}_{6}$ : Balaenoptera acutorostrata, $\mathrm{M}_{7}$ : Balaenoptera bonaerensis and $\mathrm{M}_{8}$ : Megaptera novaeangliae. $\mathrm{O}_{1}$ : Physeter macrocephalus, $\mathrm{O}_{2}$ : Delphinapterus leucas, $\mathrm{O}_{3}$ : Berardius bairdii, $\mathrm{O}_{4}$ : Ziphius cavirostris, $\mathrm{O}_{5}$ : Hyperoodon ampullatus, $\mathrm{O}_{6}$ : Orcaella brevirostris, $\mathrm{O}_{7}$ : Orcinus orca, $\mathrm{O}_{8}$ : Globicephala melas, $\mathrm{O}_{9}$ : Globicephala macrorhynchus, $\mathrm{O}_{10}$ : Pseudorca crassidens, $\mathrm{O}_{11}$ : Feresa atenuata, $\mathrm{O}_{12}$ : Peponocephala electra, $\mathrm{O}_{13}$ : Steno bredanensis, $\mathrm{O}_{14}$ : Lagenorhynchus obliquidens, $\mathrm{O}_{15}$ : Lagenorhynchus obscurus, $\mathrm{O}_{16}$ : Lagenorhynchus acutus, $\mathrm{O}_{17}$ : Lagenorhynchus australis, $\mathrm{O}_{18}$ : Grampus griseus, $\mathrm{O}_{19}$ : Tursiopus truncatus, $\mathrm{O}_{20}$ : Stenella attenuate, $\mathrm{O}_{21}$ : Stenella frontalis, $\mathrm{O}_{22}$ : Stenella longirostris, $\mathrm{O}_{23}$ : Stenella longirostris orientalis, $\mathrm{O}_{24}$ : Stenella coeruleoalba, $\mathrm{O}_{25}$ : Delphinus delphis, $\mathrm{O}_{26}$ : Delphinus capensis, $\mathrm{O}_{27}$ : Lagenodelphis hosei, $\mathrm{O}_{28}$ : Lissodelphis borealis, $\mathrm{O}_{29}$ : Celphalorhyncus comersonnii, $\mathrm{O}_{30}$ : Celphalorhyncus eutropia, $\mathrm{O}_{31}$ : Phocoenoides dalli, $\mathrm{O}_{32}$ : Phocoena phocoena and $\mathrm{O}_{33}$ : Platanista gangetica. 
in mind, a number of factors should be considered on how to approach and develop methodologies in which to investigate cetacean habitat. The following six recommendations - synthesized as a logical flow chart in Figure 5-demonstrates how future studies could become more targeted and effective in defining and describing habitat. The purpose of this outline is to progress towards a more standardized and objective approach to habitat studies. Specifically, the six recommendations developed hereafter are illustrated using selected case studies from the primary literature on both well-documented and more cryptic species to demonstrate the generality of the proposed approach.

\subsection{Identify the Rationale for Studying Habitat}

In order to describe habitat for a species or a population, the end objectives behind conducting the study firstly need to be clearly identified and addressed. Habitat characteristics should be considered on a case-by-case basis, as each species, population and location will inherently require different strategies, requirements and management considerations. An initial consideration should be the identification of research objectives, which may include 1) assisting in the development and implementation of conservation and management strategies (e.g. marine parks and reserves); 2) developing ecosystem based models; and 3) increasing the biological understanding of the animal's biology and ecology, or for mitigation purposes. For example, the habitat characteristics of the Indo-Pacific bottlenose dolphins (Tursiops aduncus) were specifically investigated with the intention to provide baseline information for a newly declared dolphin sanctuary in Adelaide, South Australia [39]. Little information existed about habitat characteristics of bottlenose dolphins in this area; hence specific habitat information was required in which to assist developing management efforts. The variety of environmental types within the declared sanctuary boundaries where dolphins were regularly sighted, were taken into consideration (e.g. ben-

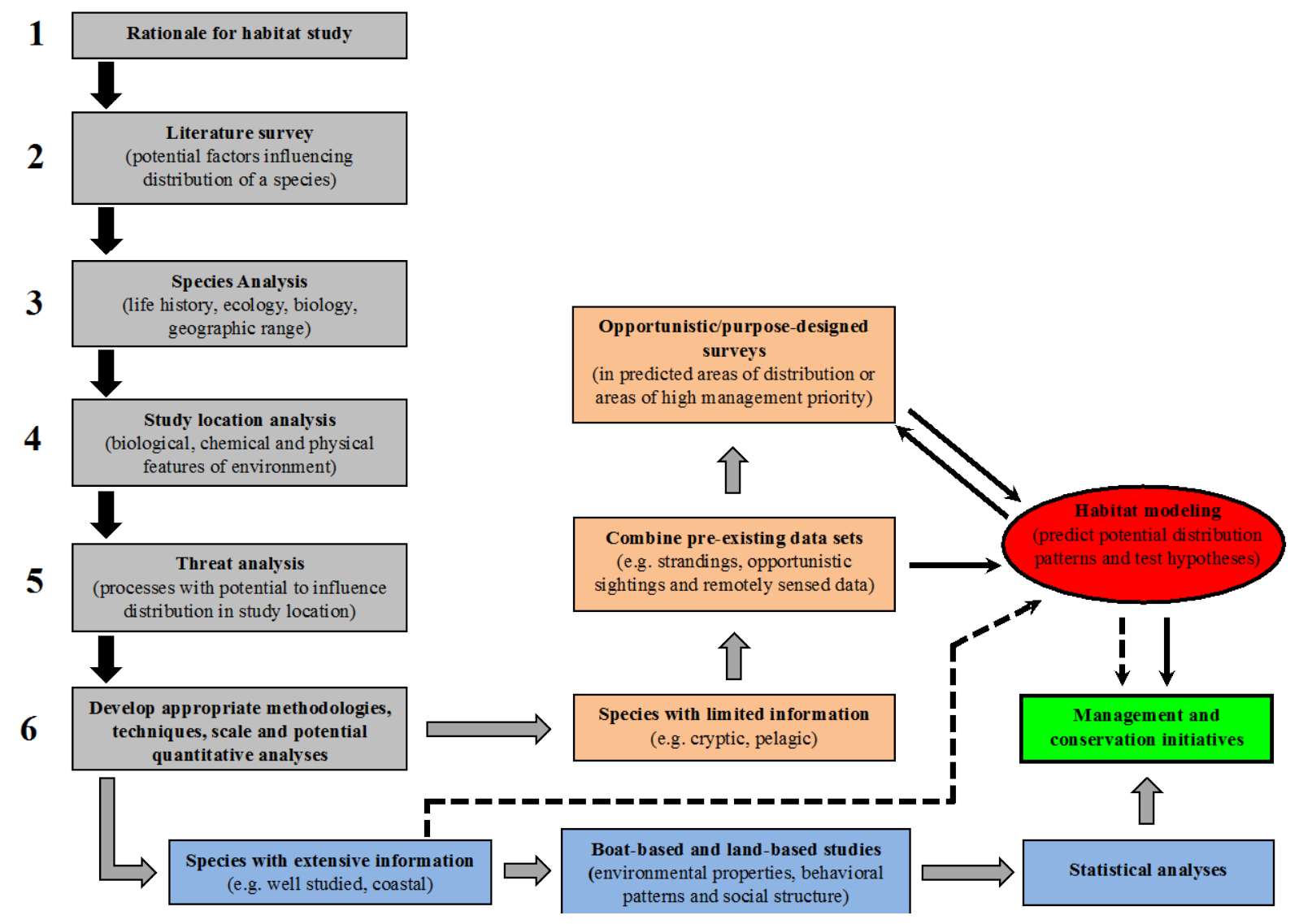

Figure 5. Logical flow chart diagram illustrating the proposed six recommendations on how future studies may become more targeted and effective in defining and describing cetacean habitat, noticeably through distinct strategies based on the status of the species in considerations, i.e. species with extensive information and/or ease of access (e.g. coastal, ubiquitous and abundant species) and species with limited information and/or difficult to study (e.g. cryptic, rare and offshore species). 
thic characteristics, exposed vs. sheltered waters). These environmental features were then incorporated into the study as each was considered to have the potential to influence dolphin presence in this area.

Similarly, [38] aimed to define critical use areas for bottlenose dolphins (Tursiops truncatus) in the Shannon estuary, Ireland, with the intention to assist management plans for a candidate Special Area of Conservation. Specific knowledge of the habitat characteristics of dolphins in this area was therefore considered crucial in developing a management strategy. In particular, the locations of dolphin encounters, were used to identify specific areas of high use, as well as any preference for areas with particular topographic features, such as depth and benthic slope. Areas identified as high use by the dolphins were then deemed "critical areas", and therefore considered to be essential to the dolphins inhabiting the estuary.

\subsection{Identify Potential Influencing Factors from the Literature}

It is critical to identify the potential factors influencing cetacean distribution, such as environmental characteristics, that have previously been identified, as well as the research methodologies that were used to do so. In some instances, there may already be considerable knowledge available. For example, numerous global studies have documented resting spinner dolphin (Stenella sp.) populations showing strong site fidelity within specific bays and reefs during daytime [55]-[57]. These studies demonstrate the consistent use of resting areas which have specific and common environmental features such as shallow, sheltered tropical bays or lagoons with sandy bottoms [58]. The identification of key environmental features provides a basis and direction in which to start the development of a habitat approach and identify the reasoning behind why these specific locations are utilized and others are not. In contrast, for those rarely sighted and data deficient species, information or potential habitat factors may be significantly lacking. In some cases only broad distribution ranges noted by a species synopsis or report may be available [5] [59]. This paucity of information can initially hinder the development of a habitat approach. However, general information about specific oceanographic occurrences or the oceanic waters within an animal's broad distribution presumed range may offer some place in which to start thinking about influencing habitat factors. Ultimately, the findings and level of information available from this type of review will assist to structure the scale and range of focus of the study.

\subsection{Species Analysis}

An essential part in approaching habitat is an assessment of the life history, ecology and biology of the species in question. Therefore species need to be considered on an individual basis. Factors such as geographic range, distribution, motion behavior and migrational patterns, home range and site fidelity need to be incorporated into the study. For rarely encountered and cryptic species, information may be limited or difficult to obtain. For example, insights into the biology, geographic range and distribution of species such as the beaked whales (family Ziphiidae) have often only been established through brief encounters and stranding occurrences [60]. This paucity about a species biological and ecological requirements allows us to then only assume those potential important factors such as geographic range. In contrast, we know a lot about some species specific movement patterns such as the Southern right whale (Eubalaena australis). Populations of this species annually migrate in the austral winter from southern Antarctic feeding grounds to sheltered waters on the Southern Australian coastline for calving [61]. The occurrence of these migration events, therefore allows a more systematic approach to be taken, as we can predict where these animals are going to occur at certain times of the year. Furthermore, we can also potentially assume their use of these areas, for example for calving.

In addition, the differing life strategies and diurnal behavioral patterns should also be considered [7]. A species life history can potentially provide insight into surrounding environmental features, as adaptations are potentially linked and influenced by it [62]. Possible inclusions for this review might include: feeding strategies, calving intervals, resting patterns and group size. In this context, spinner dolphins (Stenella sp.), are considered to have a unique life history strategy, in that some populations rest during daylight hours and feed offshore at night in the mesopelgic zone [63]. Similarly, bottlenose dolphins (Tursiops sp.) often engage in location specific foraging tactics and techniques (e.g. [64]-[66]).

\subsection{Location Analysis}

An analysis of the potential study location needs to be conducted to identify what environmental factors present 
in the area should be addressed. More specifically, the general nature of the study area's physical features/properties needs to be identified, e.g. estuary, gulf, bay or reef, exposed open ocean vs. sheltered waters. In addition, the topography, bathymetry, substrate type and the presence of islands, reefs, submarine canyons and ice cover within the environment should also be considered as potentially influencing habitat factors. Once the key features of the environment have been identified, those obvious oceanographic features and phenomena, specific to the area can then be included into the assessment. For example, water temperature, depth, salinity, turbidity, the presence and depth of a thermocline, current direction and intensity, eddies, current flow, upwelling events, primary productivity and the seasonal fluctuations of these environmental characteristics. Additionally, anthropogenic presence, predation pressure and resource availability need to be considered.

In this context, a variety of environment types had been noted to occur within the Adelaide Dolphin Sanctuary, South Australia [67]. Preliminary investigations indicated that bottlenose dolphins were frequently sighted utilizing specific areas within these different environment types. The sanctuary contained 2 distinctly different physical environments (e.g. open waters with seagrass beds and shallow, sheltered waters with bare, sandy substrate) which also potentially caused variations in the oceanographic occurrences. Therefore, within the current study plan the physical environmental features and oceanographic parameters (including seagrass presence, sheltered estuarine versus exposed gulf waters, water temperature, depth, salinity, turbidity and dissolved oxygen) considered to influence dolphin presence the most, or be important to specific life history strategies (e.g. feeding, calving) were taken into consideration as part of the survey plan. This inclusion of a wide spectrum of physical, chemical and biological environmental features such as these listed above will therefore enable a thorough investigation into those abiotic and biotic potential habitat drivers.

\subsection{Threat Analysis}

Additional factors and threats present in the marine environment should also be considered within the development of a comprehensive habitat approach. This inclusion will assist in identifying whether the presence of a threatening process drives the animal's distribution. Ultimately, this will influence how habitat is described. A study investigating the influence of repeated vessel exposure on a resident population of bottlenose dolphins (Tursiops sp.) in Shark Bay, Western Australia, then suggested that over time the repeated presence of vessels potentially could affect dolphin abundance, and as a result the habitat used [34]. Although this study did not specifically focus upon describing habitat, it demonstrated how anthropogenic impacts can potentially shift or alter the way animals distribute themselves within their surrounding environment if exposed to threats. Similarly, biological threats such as the predation of sharks also have the potential to influence distribution and ultimately the habitat used [31]. Therefore, threats to potentially include in a habitat approach are those that have the potential to affect and alter distribution. Impacts such as predator presence, pollution, drives hunts, tourism activities, commercial and artisanal hunting, fisheries, habitat degradation and climate change effects (e.g. water temperature change over time, receding ice cover) could be considered to impact distribution on an immediate, short-term or long-term level.

\subsection{Developing Appropriate Methodologies and Techniques}

The five previous considerations discussed above have identified context (in terms of objectives, species and location) and a list of factors, which should be considered within the development of a sound and objective approach to researching and studying cetacean habitat. This background information enables the selected factors to be appropriately adapted in terms of spatial and temporal scale, species biology, region and current threats. When combined with the appropriate methodologies and techniques the information gained will provide a more detailed synoptic assessment of cetacean habitat, which is therefore more targeted and applicable to potential management initiatives. However, it is considered that the suitable combination of these will ultimately begin to provide an initial insight into any potential animal and environmental relationships. Currently, many methodologies, techniques and quantitative analyses (e.g. [2] [68]-[71]) are available for application within cetacean specific research. However, these can be incorporated within a cetacean habitat approach.

In this context, the following are innovative examples of some of the ways in which cetacean habitat studies could be progressed and techniques implemented. However, this approach is not limited to these, and they are provided for illustrative purposes. The focus and implementation of methodologies and technologies will differ according to the logistics of the study location (e.g. coastal vs. offshore). Currently, many have been developed 
to assist in overcoming logistics, particularly when investigating cetaceans in the open ocean. For example, modern technologies such as remote sensing imagery, Argo floats, gliders and animal borne sensors (e.g. [32] [72]-[75]) can provide some information about the biogeographical range of cetaceans as well as open new perspectives into a detailed understanding of the vertical structure of the water column (Figure 6). When coupled with distribution patterns, for example, this oceanographic information could be used to provide insights into the potential mechanisms linking ocean processes, whales and their prey [76] [77].
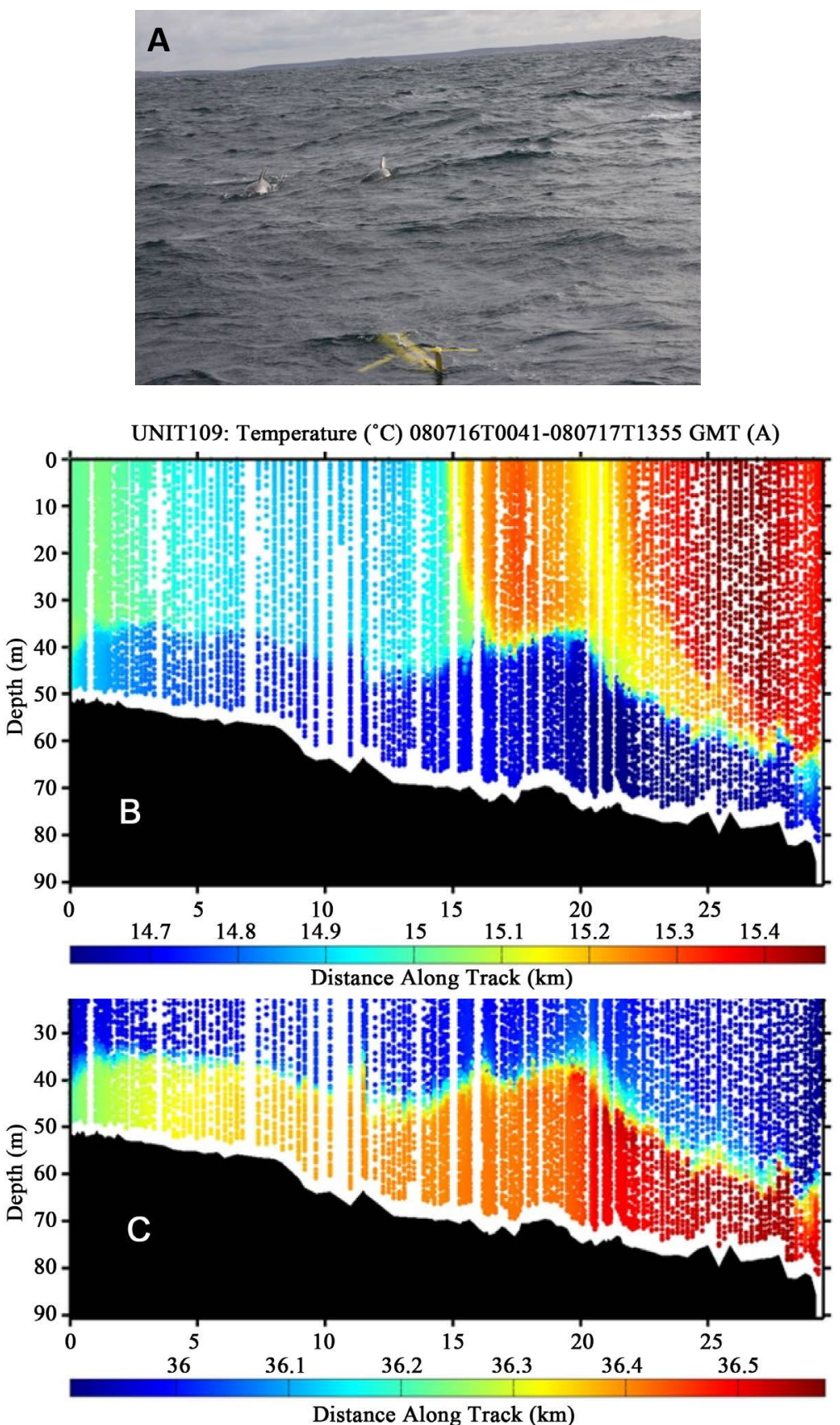

Figure 6. Modern technologies, here a Sloccum gliders deployed off Kangaroo Island (South Australia) being escorted by two bottlenose dolphins (Tursiops sp.; (A)), have the potential to assist in the collection of valuable cetacean habitat data, such as highresolution temperature (B) and salinity (C) structures. Image credit: South Australian Marine Integrated Observing System, SAIMOS [75]. 
In addition, oceanographic information has also the potential to complement data collected through opportunistic sightings, or help to correlate sighting locations, particularly for rarely sighted offshore and deep diving species. More specifically, this information may be useful, particularly for species, spending majority of their time for example, below the surface feeding such as sperm whales (Physeter microcephalus; [78]). Furthermore, as well as providing information about potential habitat correlations, these technologies can offer some insight into behavioral patterns. For example, [74] investigated the movement patterns of Blainville's beaked whales (Mesoplodon densirostris) off the coast of Hawaii using Argos-linked satellite tags. Additionally, the use of such methodologies and technologies in conjunction with in situ measurements, correlated with behavioural and social structure data can also potentially start to provide insight into cetacean ecology and life histories.

It is also stressed that the use of technologies in the field may also be complemented by, baseline cetacean habitat information gathered through the application of pre-existing data sets, particularly those gathered long term. When complemented with oceanographic information, gathered through the use of technologies or in situ, these have the potential to be of benefit to pre-existing data sets consisting of cetacean sightings and distribution patterns [79] [80]. Additionally, pre-existing data sets of species specific distributions have the potential to provide much insight into distribution in the way of being used as predictor tools for distribution (e.g. [2] [71] [81]), which ultimately can help focus a study for a specific species or location. Additionally, these can now be combined with freely accessed oceanographic datasets through ocean portals, therefore it is possible to conduct preliminary studies based on all pre-existing data.

\section{Conclusion}

Given the difficulty and complexity of adequately understanding the meaning of habitat for cetaceans, the development of a sound approach incorporating suitable techniques and methodologies is critical to enable the quantification of appropriate variables. Understanding the influences and the inter-relationships between cetaceans and their surrounding environment will not only greatly improve our understanding, but also ultimately allow us the ability to develop targeted and more effective mitigation and conservation measures.

\section{Acknowledgements}

The authors wish to thank C. Chapperon for providing comment on an earlier draft of this paper. This research was supported under Australian Research Council's Discovery Projects funding scheme (projects number DP0664681 and DP0988554). Professor Seuront is the recipient of an Australian Professional Fellowship (project number DP0988554).

\section{References}

[1] Freitas, C., Kovac, K.M., Lydersen, C. and Ims, R.A. (2008) A Novel Method for Quantifying Habitat Selection and Predicting Habitat Use. Journal of Applied Ecology, 45, 1213-1220.

[2] Redfern, J.V., Ferguson, M.C., Becker, E.A., Hyrenbach, K.D., Good, C., Barlow, J., Kaschner, K., Baumgartner, M.F., Forney, K.A., Balance, L.T., Fauchald, P., Halpin, P., Hamazaki, T., Pershing, A.J., Qian, S.S., Read, A., Reilly, S.B., Torres, L. and Werner, L. (2006) Techniques for Cetacean-Habitat Modelling. Marine Ecology Progress Series, 310, 271-295. http://dx.doi.org/10.3354/meps310271

[3] Krausman, P.R. (1999) Some Basic Principles of Habitat Use. In: Launchbaugh, K.L., Sanders, K.D. and Mosley, J.L., Eds., Grazing Behaviour of Livestock and Wildlife, Idaho Forest, Wildlife and Range Exp. Sta. Bull. No. 70, University of Idaho, Moscow, ID, 85-90.

[4] Hall, L.S., Krausmann, P.R. and Morrison, M.L. (1997) The Habitat Concept and a Plea for Standard Terminology. Wildlife Society Bulletin, 25, 173-182.

[5] Banister, J.L., Kemper, C.M. and Warneke, R.M. (1996) The Action Plan for Australian Cetaceans. Australian Nature Conservation Agency, Canberra.

[6] Reeves, R.R., Smith, R.D., Crespo, E.A. and Notarbartolo-di-Sciara, G. (2003) Dolphins, Whales and Porpoises: 2002-2010 Conservation Action Plan for the World’s Cetaceans. IUCN/SSC Cetacean Specialist Group, IUCN, Gland, Switzerland and Cambridge, UK, 139. http://dx.doi.org/10.2305/iucn.ch.2003.ssc-ap.2.en

[7] Hoyt, E. (2005) Marine Protected Areas for Whales, Dolphins and Porpoises: A Worldwide Handbook for Cetacean Habitat Conservation. Earthscan, London.

[8] Bearzi, G., Azzellino, A., Politi, E., Costa, M. and Bastianni, M. (2008) Influence of Seasonal Forcing on Habitat Use 
of Bottlenose Dolphins Tursiops truncatus in the Northern Adriatic Sea. Ocean Science Journal, 43, 175-182. http://dx.doi.org/10.1007/BF03029922

[9] Perrin, W.F. (2009) Geographic Variation. In: Perrin, W.F., Wursig, B. and Thewissen, J.G.M., Eds., Marine Mammal Encyclopaedia, 2nd Edition, Academic Press, San Diego, 492-498. http://dx.doi.org/10.1016/b978-0-12-373553-9.00116-4

[10] Kearney, M. (2006) Habitat, Environment and Niche: What Are We Modelling? Oikos, 115, 186-191. http://dx.doi.org/10.1111/j.2006.0030-1299.14908.x

[11] Mitchell, S.C. (2005) How Useful Is the Concept of Habitat?-A Critique. Oikos, 110, 634-638. http://dx.doi.org/10.1111/j.0030-1299.2005.13810.x

[12] Kolasa, J. and Pickett, S.T. (1993) Ecological Heterogeneity. Springer-Verlag, New York.

[13] Ricklefs, R.E. (1993) The Economy of Nature: A Textbook in Basic Ecology. W.H. Freeman and Company, New York.

[14] International Union for the Conservation of Nature (2009) The IUCN Red List of Threatened Species. http://www.redlist.org/

[15] Gaskin, D.E. (1968) Distribution of Delphinidae (Cetacea) in Relation to Sea Surface Temperatures off Eastern and Southern New Zealand. New Zealand Journal of Marine and Freshwater Research, 2, 527-534. http://dx.doi.org/10.1080/00288330.1968.9515253

[16] Wilson, B., Thompson, P.M. and Hammond, P.S. (1997) Habitat Use by the Bottlenose Dolphins: Seasonal Distribution and Stratified Movement Patterns in the Moray Firth, Scotland. Journal of Applied Ecology, 34, 1365-1374. http://dx.doi.org/10.2307/2405254

[17] Selzer, L.A. and Payne, M.P. (1988) The Distribution of White-Sided (Lagenorhynchus acutus) and Common Dolphins (Delphinus delphis) vs. Environmental Features of the Continental Shelf of the Northeastern Unites States. Marine Mammal Science, 4, 141-153. http://dx.doi.org/10.1111/j.1748-7692.1988.tb00194.x

[18] Bräger, S., Harraway, J.A. and Manly, B.F.J. (2003) Habitat Selection in a Coastal Dolphin Species (Cephalorhynchus hectori). Marine Biology, 143, 233-244. http://dx.doi.org/10.1007/s00227-003-1068-x

[19] Kiszka, J., Macleod, K., Van Canneyt, O., Walker, D. and Ridoux, V. (2007) Distribution, Encounter Rates, and Habitat Characteristics of Toothed Cetaceans in the Bay of Biscay and Adjacent Waters from Platform-of-Opportunity. ICES, 64, 1-11. http://dx.doi.org/10.1093/icesjms/fsm067

[20] Ballance, L.T., Pitman, R.L. and Fielder, P.C. (2006) Oceanographic Influences on Seabirds and Cetaceans of the Eastern Tropical Pacific: A Review. Progress in Oceanography, 69, 360-390. http://dx.doi.org/10.1016/j.pocean.2006.03.013

[21] Leatherwood, S., Peters, C.B. and Clarke, J.T. (1984) Observations of Cetaceans in the Northern Indian Ocean Sanctuary, November 1980-May 1983. Reports of the International Whaling Committee, 34, 509-520.

[22] Forney, K.A. and Barlow, J. (1998) Seasonal Patterns in the Abundance and Distribution of California Cetaceans, 1991-92. Marine Mammal Science, 14, 460-489. http://dx.doi.org/10.1111/j.1748-7692.1998.tb00737.x

[23] Moore, S.E. (2000) Variability of Cetacean Distribution and Habitat Selection in the Alaskan Arctic, Autumn $1982-91$. Arctic, 53, 448-460. http://dx.doi.org/10.14430/arctic874

[24] Thiele, D., Chester, E.T. and Gill, P.C. (2000) Cetacean Distribution off Eastern Antarctica (80-150 E) during the Austral Summer of 1995/1996. Deep-Sea Research Part II, 47, 2543-2572. http://dx.doi.org/10.1016/S0967-0645(00)00035-7

[25] Weir, C.R., Pollock, C., Cronin, C. and Taylor, S. (2001) Cetaceans of the Atlantic Frontier, North and West of Scotland. Continental Shelf Research, 21, 1047-1071. http://dx.doi.org/10.1016/S0278-4343(00)00124-2

[26] Miller, C. (2007) Current State of Knowledge of Cetacean Threats, Diversity, and Habitats in the Pacific Islands Region. WDCS Australasia Inc., Adelaide.

[27] Heithaus, M.R., Marshall, G.J., Buhleier, B.M. and Dill, L.M. (2001) Employing Crittercam to Study Habitat Use and Behavior of Large Sharks. Marine Ecology Progress Series, 209, 307-310. http://dx.doi.org/10.3354/meps209307

[28] Weinrich, M. (1998) Early Experience in Habitat Choice by Humpback Whales (Megaptera novaeangliae). Journal of Mammalogy, 79, 163-170. http://dx.doi.org/10.2307/1382851

[29] Montero, R. and Arechavaleta, M. (1996) Distribution Patterns, Relationships between Depth, Sea Surface Temperature, and Habitat Use of Short-Finned Pilot Whales South-West of Tenerife. European Research on Cetaceans, 10, 193-197.

[30] Jenner, K.C.S., Jenner, M.N.M. and McCabe, K.A. (2001) Geographical and Temporal Movements of Humpback Whales in Western Australian Waters. AAPEA Journal, 2001, 749-765. 
[31] Heithaus, M.R. and Dill, L.M. (2002) Food Availability and Tiger Shark Predation Risk Influence Bottlenose Dolphin Habitat Use. Ecology, 83, 480-491. http://dx.doi.org/10.1890/0012-9658(2002)083[0480:FAATSP]2.0.CO;2

[32] Hastie, G.D., Wilson, B., Wilson, L.J. and Parsons, K.M. (2004) Functional Mechanisms Underlying Cetacean Distribution Patterns: Hotspots for Bottlenose Dolphins Are Linked to Foraging. Marine Biology, 144, 397-403. http://dx.doi.org/10.1007/s00227-003-1195-4

[33] Watson-Capps, J.J. and Mann, J. (2005) The Effects of Aquaculture on Bottlenose Dolphins (Tursiops sp.) Ranging in Shark Bay, Western Australia. Biological Conservation, 124, 519-526. http://dx.doi.org/10.1016/j.biocon.2005.03.001

[34] Bejder, L., Samuels, A., Mann, J., Whitehead, H., Gales, N., Connor, R., Heithaus, M., Watson-Capps, J., Flaherty, C. and Krutzen, M. (2006) Decline in Relative Abundance of Bottlenose Dolphins Exposed to Long-Term Disturbance. Conservation Biology, 20, 1791-1798. http://dx.doi.org/10.1111/j.1523-1739.2006.00540.x

[35] Riberio, S., Viddi, F.A., Cordeiro, J.L. and Freitas, T.R.O. (2007) Fine-Scale Habitat Selection of Chilean Dolphins (Cephalorhynchus eutropia): Interactions with Aquaculture Activities in Southern Chiloé Island, Chile. Journal of the Marine Biological Association of the United Kingdom, 87, 119-128. http://dx.doi.org/10.1017/S0025315407051594

[36] Miller, C. and Cribb, N. (2009) Describing Cetacean Habitat in Australian Waters. La Mer, 46, 77-84.

[37] Maze, K.S. and Wursig, B. (1999) Bottlenose Dolphins of San Luis Pass, Texas: Occurrence Patterns, Site-Fidelity and Habitat Use. Aquatic Mammals, 25, 90-103.

[38] Ingram, S.N. and Rogan, E. (2002) Identifying Critical Areas and Habitat Preferences of Bottlenose Dolphins Tursiops truncatus. Marine Ecology Progress Series, 244, 247-255. http://dx.doi.org/10.3354/meps244247

[39] Cribb, N., Miller, C. and Seuront, L. (2008) Assessment of Bottlenose Dolphin (Tursiops aduncus) Habitat Characteristics in the Estuarine Waters of the Adelaide Dolphin Sanctuary, South Australia. The Journal of Marine Animals and Their Ecology, 1, 6-8.

[40] Miller, C. and Baltz, D.M. (2009) Environmental Characterization of Seasonal Trends and Foraging Habitat of Bottlenose Dolphins (Tursiops truncatus) in the Northern Gulf of Mexico Bays. Fisheries Bulletin, 108, 79-86.

[41] Laran, S. and Drouot-Dulau, V. (2007) Seasonal Variation of Striped Dolphins, Fin- and Sperm Whales’ Abundance in the Ligurian Sea (Mediterranean Sea). Journal of the Marine Biological Association of the United Kingdom, 87, 345352. http://dx.doi.org/10.1017/S0025315407054719

[42] Baumgartner, M.F. (1997) The Distribution of Risso’s Dolphin (Grampus griseus) with Respect to the Physiography of the Northern Gulf of Mexico. Marine Mammal Science, 13, 614-638. http://dx.doi.org/10.1111/j.1748-7692.1997.tb00087.x

[43] Keiper, C.A., Ainley, D.G., Allen, S.G. and Harvey, J.T. (2005) Marine Mammal Occurrence and Ocean Climate off Central California, 1986 to 1994 and 1997 to 1999. Marine Ecology Progress Series, 289, 285-306. http://dx.doi.org/10.3354/meps289285

[44] Danielwicz, D., Secchi, E.R., Ott, P.H., Moreno, I.B., Bassoi, M. and Borges-Martins, M. (2009) Habitat Use Patterns of Franciscana Dolphins (Pontoporia blainvillei) off Southern Brazil in Relation to Water Depth. Journal of the Marine Biological Association of the United Kingdom, 89, 943-949. http://dx.doi.org/10.1017/S002531540900054X

[45] Kenney, R.D. and Winn, H.E. (1986) Cetacean High-Use Habitats of the Northeast United States Continental Shelf. Fisheries Bulletin, 84, 345-357.

[46] Gregr, E.J., Baumgartner, M.F., Laidre, K.L. and Palacios, D.M. (2013) Marine Mammal Habitat Models Come of Age: The Emergence of Ecological and Management Relevance. Endangered Species Research, 22, 205-212. http://dx.doi.org/10.3354/esr00476

[47] Canadas, A., Fortuna, C.M. and Hammond, P.S. (2006) Modelling Techniques to Investigate the Impact of Changes in Habitat on Cetacean Distribution and Abundance. Proceedings of the 58th International Whaling Commission, St. Kitts \& Nevis, 16-20 June 2006, 21.

[48] Palacios, D.M., Baumgartner, M.F., Laidre, K.L. and Gregr, E.J. (2013) Beyond Correlation: Integrating Environmentally and Behaviourally Mediated Processed in Models of Marine Mammal Distributions. Endangered Species Research, 22, 191-203. http://dx.doi.org/10.3354/esr00558

[49] Ferguson, M.C., Barlow, J., Reilly, S.B. and Gerrodette, T. (2006) Predicting Cuvier's (Ziphius cavirostris) and Mesoplodon Beaked Whale Population Density from Habitat Characteristics in the Eastern Tropical Pacific Ocean. Journal of Cetacean Research and Management, 7, 287-299.

[50] Pershing, A.J., Record, N.R., Monger, B.C., Mayo, C.A., Brown, M.W., Cole, T.V.N., Kenney, R.D., Pendleton, D.E. and Woodard, L.A. (2009) Model-Based Estimates of Right Whale Habitat Use in the Gulf of Maine. Marine Ecology Progress Series, 378, 245-257. http://dx.doi.org/10.3354/meps07829

[51] Johnston, D.W., Chapla, M.E., Williams, L.E. and Mattila, D.K. (2007) Identification of Humpback Whale Megaptera novaeangliae Wintering Habitat in the Northwestern Hawaiian Islands Using Spatial Habitat Modeling. Endangered 
Species Research, 3, 249-257.

[52] Praca, E. and Gannier, A. (2008) Ecological Niches of Three Teuthophageous Odontocetes in the Northwestern Mediterranean Sea. Ocean Science, 4, 49-59. http://dx.doi.org/10.5194/os-4-49-2008

[53] Soldevilla, M.S., Wiggins, S.M., Hildebrand, J.A., Oleson, E.M. and Ferguson, M.C. (2011) Risso's and Pacific White-Sided Dolphin Habitat Modelling from Passive Acoustic Monitoring. Marine Ecology Progress Series, 423, 247-260. http://dx.doi.org/10.3354/meps08927

[54] Vierling, K.T., Vierling, L.A., Gould, W.A., Martinuzzi, S. and Clawges, R.M. (2008) Lidar: Shedding New Light on Habitat Characterization and Modeling. Frontiers in Ecology, 6, 90-98. http://dx.doi.org/10.1890/070001

[55] Karczmarski, L., Wursig, B., Gailey, G., Larson, K.W. and Vandelip, C. (2005) Spinner Dolphins in a Remote Hawaiian Atoll: Social Grouping and Population Structure. Behavioral Ecology, 16, 675-685. http://dx.doi.org/10.1093/beheco/ari028

[56] Gannier, A. and Petiau, E. (2006) Environmental Variables Affecting the Residence of Spinner Dolphins (Stenella longirostris) in a Bay of Tahiti (French Polynesia). Aquatic Mammals, 32, 202-211. http://dx.doi.org/10.1578/AM.32.2.2006.202

[57] Notarbartolo-di-Sciara, G., Hanafy, M.H., Fouda, M.M., Afifi, A. and Costa, A. (2009) Spinner Dolphin (Stenella longirostris) Resting Habitat in Samadai Reef (Egypt, Red Sea) Protected through Tourism Management. Journal of the Marine Biological Association of the United Kingdom, 89, 211-216. http://dx.doi.org/10.1017/S0025315408002221

[58] Cribb, N., Miller, C. and Seuront, L. (2012) Indo-Pacific Bottlenose Dolphin (Tursiops aduncus) Habitat Preference in a Heterogeneous, Urban, Coastal Environment. Aquatic Biosystems, 9, 3-11. http://dx.doi.org/10.1186/2046-9063-9-3

[59] Ross, G.J.B. (2006) Review of the Conservation Status of Australia's Smaller Whales and Dolphins. Australian Government.

[60] MacLeod, C.D., Perrin, W.F., Pitman, R., Barlow, J., Balance, L., D’Amico, A., Gerrodette, T., Joyce, G., Mullin, K.D., Palka, D.L. and Waring, G.T. (2006) Known and Inferred Distributions of Beaked Whale Species (Cetacea: Ziphiidae). Journal of Cetacean Research and Management Special Issue, 7, 271-286.

[61] Pirzl, R. (2008) Spatial Ecology of E. australis: Habitat Selection at Multiple Scales. Ph.D. Thesis, Deakin University, Victoria.

[62] Chivers, S.J. (2009) Cetacean Life-History. In: Perrin, W.F., Wursig, B. and Thewissen, J.G.M., Eds., Marine Mammal Encyclopedia, Academic Press, San Diego, 492-498. http://dx.doi.org/10.1016/b978-0-12-373553-9.00055-9

[63] Norris, K.S. and Dohl, T.P. (1980) Behavior of the Hawaiian Spinner Dolphin, Stenella longirostris. Fisheries Bulletin, 77, 821-849.

[64] Torres, L.G. and Read, A.J. (2009) Where to Catch a Fish? The Influence of Foraging Tactics on the Ecology of Bottlenose Dolphins (Tursiops truncatus) in Florida Bay, Florida. Marine Mammal Science, 25, 797-815. http://dx.doi.org/10.1111/j.1748-7692.2009.00297.x

[65] Duffy-Echevariia, E., Connor, R.C. and St. Aubin, D.J. (2008) Observations of Strand-Feeding Behaviour Bottlenose Dolphins (Tursiops truncatus) in Bull Creek, South Carolina. Marine Mammal Science, 24, 202-206. http://dx.doi.org/10.1111/j.1748-7692.2007.00151.x

[66] Smolker, R., Richards, A., Connor, R.C., Mann, J. and Berggren, P. (1997) Sponge Carrying by Dolphins (Delphinidae, Tursiops sp.): A Foraging Specialization Involving Tool Use? Ethology, 103, 454-465. http://dx.doi.org/10.1111/j.1439-0310.1997.tb00160.x

[67] Department of Environment, Water and Natural Resources (2013) The Adelaide Dolphin Sanctuary. http://www.naturalresources.sa.gov.au/adelaidemtloftyranges/coast-and-marine/dolphin-sanctuary

[68] Mann, J. (1999) Behavioural Sampling Methods for Cetaceans: A Review and Critique. Marine Mammal Science, 15, 102-122. http://dx.doi.org/10.1111/j.1748-7692.1999.tb00784.x

[69] Evans, P.G.H. and Hammond, P.S. (2004) Monitoring Cetaceans in European Waters. Mammal Review, 34, $131-156$. http://dx.doi.org/10.1046/j.0305-1838.2003.00027.x

[70] Forney, K.A. and Wade, P.R. (2006) Worldwide Distribution and Abundance of Killer Whales. In: Estes, J.A., Demaster, D.P., Doak, D.F., Williams, T.M. and Brownell Jr., R.L., Eds., Whales, Whaling and Ocean Ecosystems, University of California Press, Berkeley, CA, 145-162.

[71] Kaschner, K., Watson, R., Trites, A.W. and Pauly, D. (2006) Mapping World-Wide Distributions of Marine Mammal Species Using a Relative Environmental Suitability (RES) Model. Marine Ecology Progress Series, 316, 285-310. http://dx.doi.org/10.3354/meps316285

[72] McMahon, C.R., Autret, E., Houghton, J.D.R., Lovell, P., Myers, A.E. and Hays, G.C. (2005) Animal-Borne Sensors Successfully Capture the Real-Time Thermal Properties of Ocean Basins. Limnology and Oceanography: Methods, 3, 392-398. http://dx.doi.org/10.4319/lom.2005.3.392 
[73] Boehme, L., Lovell, P., Biuw, M., Roquet, F., Nicholson, J., Thorpe, S.E., Meredith, M.P. and Fedak, M. (2009) Technical Note: Animal-Borne CTD-Satellite Relay Data Loggers for Real-Time Oceanographic Data Collection. Ocean Science, 5, 685-695. http://dx.doi.org/10.5194/os-5-685-2009

[74] Schorr, G.S., Baird, R.W., Hanson, M.B., Webster, D.L., McSweeney, D.J. and Andrews, R.D. (2009) Movements of Satellite-Tagged Blainville’s Beaked Whales off the Island of Hawaii. Endangered Species Research, 10, 203-213. http://dx.doi.org/10.3354/esr00229

[75] Integrated Marine Observing System (2010) Argo Floats. http://imos.org.au/argo.html

[76] Fiedler, P.C., Reilly, S.B., Hewitt, R.P., Demer, D., Philbrick, V.A., Smith, S., Armstrong, W., Croll, D.A., Tershy, B.R. and Mate, B.R. (1998) Blue Whale Habitat and Prey in the California Channel Islands. Deep-Sea Research Part II, 45, 1781-1801. http://dx.doi.org/10.1016/S0967-0645(98)80017-9

[77] Tynan, C.T., Ainley, D.G., Barth, J.A., Cowles, T.J., Pierce, S.D. and Spear, L.B. (2005) Cetacean Distributions Relative to Ocean Processes in the Northern California Current System. Deep-Sea Research Part II, 52, 145-167. http://dx.doi.org/10.1016/j.dsr2.2004.09.024

[78] Watwood, S.L., Miller, P.J.O., Johnson, M., Madsen, P.T. and Tyack, P.L. (2006) Deep-Diving Foraging Behaviour of Sperm Whales (Physeter macrocephalus). Journal of Animal Ecology, 75, 814-825. http://dx.doi.org/10.1111/j.1365-2656.2006.01101.x

[79] Maury, M.F. (1852) Wind and Current Charts. Whale Sheets Number 1 to 4, Series F. United States Hydrographical Office, Washington DC.

[80] Townsend, C.H. (1935) The Distribution of Certain Whales as Shown by Logbook Records of American Whalerships. New York Zoological Society, 19, 1-50.

[81] Jacquet, J. and Whitehead, H. (1996) Scale-Dependent Correlation Distribution with Environmental Features and Productivity in the South Pacific. Marine Ecology Progress Series, 135, 1-9. http://dx.doi.org/10.3354/meps135001

[82] Udvardy, M.F.D. (1959) Notes on the Ecological Concepts of Habitat, Biotope and Niche. Ecology, 40, 725-727. http://dx.doi.org/10.2307/1929830

[83] Odum, E.P. (1963) Ecology. Holt, Rinehart \& Winston, Inc., New York.

[84] Schreiner, K.M. (1976) Critical Habitat: What It Is and Is Not. Endangered Species Bulletin, 1, 3-4.

[85] Harris, L.D. and Kangas, P. (1988) Reconsideration of the Habitat Concept. In: McCabe, R.E., Ed., Transactions of the 53rd North American Wildlife and Natural Resources Conference Wildlife and Natural Conference, Wildlife Management Institute, Washington DC, 137-144.

[86] Jones, K.M.M. and Boulding, E.G. (1999) State-Dependent Habitat Selection by an Intertidal Snail: The Costs of Selecting a Physically Stressful Microhabitat. Journal of Experimental Marine Biology and Ecology, 242, 149-177. http://dx.doi.org/10.1016/S0022-0981(99)00090-8

[87] Garshelis, D.L. (2000) Delusions in Habitat Evaluation: Measuring Use, Selection, and Importance. In: Boitani, L. and Fuller, T.K., Eds., Research Techniques in Animal Ecology: Controversies and Consequences, Columbia University Press, New York, 111-164.

[88] Morrison, M.L. (2001) A Proposed Research Emphasis to Overcome Limits of Wildlife-Habitat Relationship Studies. Journal of Wildlife Management, 65, 613-623. http://dx.doi.org/10.2307/3803012

[89] Ragen, T.J. (2005) Assessing and Managing Marine Mammal Habitat in the United States. In: Reynolds, J.E., Perrin, W.F., Reeves, R.R., Montgomery, S. and Ragen, T.J., Eds., Marine Mammal Research: Conservation beyond Crisis, The John Hopkins University Press, Baltimore, 125-162.

[90] Orth, D.J. and White, R.J. (1993) Stream Habitat Management. In: Kohler, C. and Hubert, W., Eds., Inland Fisheries Management in North America, American Fisheries Society, Bethesda, 205-230.

[91] Ray, G. and McCormick-Ray, M. (1995) Critical Habitats and Representative Systems in Marine Environments: Concepts and Procedures. In: Agardy, T., Ed., The Science of Conservation in the Coastal Zone, International Union for the Conservation of Nature and Natural Resources, Gland, Switzerland, 23-40.

[92] Harwood, J. (2001) Marine Mammals and Their Environment in the Twenty-First Century. Journal of Mammalogy, 82, 630-640. http://dx.doi.org/10.1093/jmammal/82.3.630

[93] Nybakken, J.W. (2001) Marine Biology: An Ecological Approach. 5th Edition, Benjamin Cummings, San Francisco.

[94] Connor, D.W., Allen, J.H., Golding, N., Lieberknecht, L.M., Northen, K.O. and Reker, J.B. (2003) The National Marine Habitat Classification for Britain and Ireland. Version 03.02, Introductory Text, Joint Nature Conservation Committee, Peterborough.

[95] Shane, S. (1980) Occurrence, Movements and Distribution of Bottlenose Dolphin, Tursiops truncatus, in Southern Texas. Fisheries Bulletin, 78, 593-601. 
[96] Corkeron, P.J. (1990) Aspects of the Behavioural Ecology of Inshore Dolphins Tursiops truncatus and Sousa chinensis in Moreton Bay, Australia. In: Leatherwood, S. and Reeves, R.R., Eds., The Bottlenose Dolphin, Academic Press, San Diego, 245-265.

[97] Ballance, L.T. (1992) Habitat Use Patterns and Ranges of the Bottlenose Dolphin in the Gulf of California, Mexico. Marine Mammal Science, 8, 262-274. http://dx.doi.org/10.1111/j.1748-7692.1992.tb00408.x

[98] Karczmarski, L., Cockcroft, V.G. and McLachlan, A. (2000) Habitat Use and Preferences of Indo-Pacific Humpback Dolphins Sousa chinensis in Algoa Bay, South Africa. Marine Mammal Science, 16, 65-79. http://dx.doi.org/10.1111/j.1748-7692.2000.tb00904.x

[99] Mann, J., Connor, R.C., Barre, L.M. and Heithaus, M.R. (2000) Female Reproductive Success in Bottlenose Dolphins (Tursiops sp.): Life History, Habitat, Provisioning and Group Size Effects. Behavioral Ecology, 11, 210-219. http://dx.doi.org/10.1093/beheco/11.2.210

[100] Allen, M.C., Read, A.J. and Gaudet, J. (2001) Fine Scale Habitat Selection of Foraging Bottlenose Dolphins Tursiops truncatus near Clearwater, Florida. Marine Ecology Progress Series, 222, 253-264. http://dx.doi.org/10.3354/meps222253

[101] Bejder, L. and Dawson, S. (2001) Abundance, Residency, and Habitat Utilization of Hector's Dolphins (Cephalorhynchus hectori) in Porpoise Bay, New Zealand. New Zealand Journal of Marine and Freshwater Research, 35, 277-287. http://dx.doi.org/10.1080/00288330.2001.9516998

[102] Mendes, S., Turrell, W., Lutkebohle, T. and Thompson, P. (2002) Influence of the Tidal Cycle and a Tidal Intrusion front on the Spatio-Temporal Distribution of Coastal Bottlenose Dolphins. Marine Ecology Progress Series, 239, 221229. http://dx.doi.org/10.3354/meps239221

[103] Griffin, R.B. and Griffin, N.J. (2003) Distribution, Habitat Partitioning, and Abundance of Atlantic Spotted Dolphins, Bottlenose Dolphins, and Loggerhead Sea Turtles on the Eastern Gulf of Mexico Continental Shelf. Gulf of Mexico Science, 1, 23-24.

[104] Armstrong, P., Arthur, C. and Murray, C. (2005) Migratory Bottlenose Dolphin Movements and Numbers along the Mid-Atlantic Coast and Their Correlation with Remotely Sensed Chlorophyll- $\alpha$ and Sea Surface Temperatures. Report Prepared for the Undergraduate Research Experience in Ocean and Marine Science Program, Elizabeth City State University, Elizabeth City, NC.

[105] Fogg, T.J. (2005) Dolphin Presence/Absence Probabilities on the Virginia and North Carolina Coasts as Correlated with Sea Surface Temperature and Chlorophyll- $\alpha$ Levels. Geoscience \& Remote Sensing Symposium, 3, 1608-1611. http://dx.doi.org/10.1109/igarss.2005.1526304

[106] Moreno, I.B., Zerbini, A.N., Danilewicz, D., de Oliveira Santis, M.C., Simoes-Lopes, P.C., Lailson-Brito Jr., J. and Azevedo, A.F. (2005) Distribution and Habitat Characteristics of Dolphins in the Genus Stenella (Cetacea: Delphinidae) in the Southwest Atlantic Ocean. Marine Ecology Progress Series, 300, 229-240. http://dx.doi.org/10.3354/meps300229

[107] Parra, G.J. (2005) Behavioural Ecology of Irrawady, Orcaella brevirostris (Owen in Gary, 1866), and Ind-Pacific Humpback Dolphins, Sousa chinensis (Osbeck, 1765), in Northeast Queensland, Australia: A Comparative Study. Ph.D. Thesis, James Cook University, Queensland.

[108] Viddi, F.A. and Lescrauwaet, A.K. (2005) Insights on Habitat Selection and Behavioural Patterns of Peale’s Dolphins (Lagenorhynchus australis) in the Strait of Magellan, Southern Chile. Aquatic Mammals, 31, 176-183. http://dx.doi.org/10.1578/AM.31.2.2005.176

[109] Parra, G.J. (2006) Resource Partitioning in Sympatric Delphinids: Space Use and Habitat Preferences of Australian Snubfin and Indo-Pacific Humpback Dolphins. Journal of Animal Ecology, 78, 862-874. http://dx.doi.org/10.1111/j.1365-2656.2006.01104.x

[110] Cañadas, A. and Hammond, P.S. (2008) Abundance and Habitat Preferences of the Short-Beaked Common Dolphin Delphinus delphis in the Southwestern Mediterranean: Implications for Conservation. Marine Ecology Progress Series, 4, 309-331.

[111] Goodwin, L. (2008) Diurnal and Tidal Variations in Habitat Use of the Harbour Porpoise (Phocoena phocoena) in Southwest Britain. Aquatic Mammals, 34, 44-53. http://dx.doi.org/10.1578/AM.34.1.2008.44

[112] Filby, N.E., Bossley, M., Sanderson, K.J., Martinez, E. and Stockin, K.A. (2010) Distribution and Population Demographics of Common Dolphins (Delphinus delphis) in the Gulf St. Vincent, South Australia. Aquatic Mammals, 36, $33-$ 45. http://dx.doi.org/10.1578/AM.36.1.2010.33

[113] Hooker, S.K., Whitehead, H. and Gowans, S. (1999) Marine Protected Area Design and the Spatial and Temporal Distribution of Cetaceans in a Submarine Canyon. Conservation Biology, 13, 592-602. http://dx.doi.org/10.1046/j.1523-1739.1999.98099.x

[114] Forney, K.A. (2000) Environmental Models of Cetacean Abundance: Reducing Uncertainty in Population Trends. 
Conservation Biology, 14, 1271-1286. http://dx.doi.org/10.1046/j.1523-1739.2000.99412.x

[115] Gregr, E.J. and Trites, A.W. (2001) Predictions of Critical Habitat for Five Whale Species in the Waters of Coastal British Columbia. Canadian Journal of Fisheries and Aquatic Sciences, 58, 1265-1285. http://dx.doi.org/10.1139/f01-078

[116] Hamazaki, T. (2002) Spatiotemporal Prediction Models of Cetacean Habitats in the Mid-Western North Atlantic Ocean (From Cape Hatteras, North Carolina, USA to Nova Scotia, Canada). Marine Mammal Science, 18, 920-939. http://dx.doi.org/10.1111/j.1748-7692.2002.tb01082.x

[117] Canadas, A., Sagarminaga, R., De Stephanis, R., Urquiola, E. and Hammond, P.S. (2005) Habitat Preference Modelling as a Conservation Tool: Proposals for Marine Protected Areas for Cetaceans in Southern Spanish Waters. Aquatic Conservation: Marine and Freshwater Ecosystems, 15, 495-521. http://dx.doi.org/10.1002/aqc.689

[118] Hastie, G.D., Swift, R.J., Slesser, G., Thompson, P.M. and Turrell, W.R. (2005) Environmental Models for Predicting Oceanic Dolphin Habitat in the Northeast Atlantic. ICES Journal of Marine Science, 62, 760-770. http://dx.doi.org/10.1016/j.icesjms.2005.02.004

[119] Ferguson, M.C., Barlow, J., Fiedler, P., Reilly, S.B. and Gerrodette, T. (2006b) Spatial Models of Delphinid (Delphinidae) Encounter Rate and Group Size in the Eastern Tropical Pacific Ocean. Ecological Modelling, 193, 645-662. http://dx.doi.org/10.1016/j.ecolmodel.2005.10.034

[120] Frielaender, A.S., Halpin, P.N., Qian, S.S., Lawson, G.L., Wiebe, P.H., Thiele, D. and Read, A.J. (2006) Whale Distribution in Relation to Prey Abundance and Oceanographic Processes in the Shelf Waters of the Western Antarctic Peninsula. Marine Ecology Progress Series, 317, 297-310. http://dx.doi.org/10.3354/meps317297

[121] Goetz, K.T., Rugh, D.J., Read, A.J. and Hobbs, R.C. (2007) Habitat Use in a Marine Ecosystem: Beluga Delphinapterus leucas in Cook Inlet, Alaska. Marine Ecology Progress Series, 330, 247-256. http://dx.doi.org/10.3354/meps330247

[122] Certain, G., Ridoux, O., van Canneyt, O. and Bretagnolle, V. (2008) Delphinid Spatial Distribution and Abundance Estimates over the Shelf of the Bay of Biscay. ICES Journal of Marine Science, 65, 656-666. http://dx.doi.org/10.1093/icesjms/fsn046

[123] De Stephanis, R., Cornulier, T., Verbough, P., Sierra, J.S., Gimeno, N.P. and Guinet, C. (2008) Summer Spatial Distribution of Cetaceans in the Strait of Gibraltar in Relation to the Oceanographic Context. Marine Ecology Progress Series, 353, 275-288. http://dx.doi.org/10.3354/meps07164

[124] Gómez De Segura, A., Hammond, P.S. and Raga, J.A. (2008) Influence of Environmental Factors on Small Cetacean Distribution in the Spanish Mediterranean. Journal of the Marine Biological Association of the United Kingdom, 88, 1185-1192. http://dx.doi.org/10.1017/S0025315408000386

[125] Panigada, S., Zanardelli, M., MacKenzie, M., Donovan, C., Mélin, F. and Hammond, P.S. (2008) Modelling Habitat Preferences for Fin Whales and Striped Dolphins in the Pelagos Sanctuary (Western Mediterranean Sea) with Physiographic and Remote Sensing Variables. Remote Sensing of Environment, 112, 3400-3412. http://dx.doi.org/10.1016/j.rse.2007.11.017

[126] Redfern, J.V., Barlow, J., Balance, L.T., Gerrodette, T. and Becker, E.A. (2008) Absence of Scale Dependence in Dolphin-Habitat Models for the Eastern Tropical Pacific Ocean. Marine Ecology Progress Series, 363, 1-14. http://dx.doi.org/10.3354/meps07495

[127] Skov, H., Gunnlaugsson, T., Budgell, W.P., Horne, J., Nøttestad, L., Olsen, E., Søiland, H., Víkingsson, G. and Waring, G. (2007) Small-Scale Spatial Variability of Sperm and Sei Whales in Relation to Oceanographic Features along the Mid-Atlantic Ridge. Deep-Sea Research II, 55, 254-268. http://dx.doi.org/10.1016/j.dsr2.2007.09.020

[128] Torres, L.G., Read, A.J. and Halpin, P. (2008) Fine-Scale Habitat Modeling of a Top Marine Predator: Do Prey Data Improve Predicative Capacity? Ecological Applications, 18, 1702-1717. http://dx.doi.org/10.1890/07-1455.1

[129] Bailey, H. and Thompson, P.M. (2009) Using Marine Mammal Habitat Modelling to Identify Priority Conservation Zones within a Marine Protected Area. Marine Ecology Progress Series, 378, 279-287. http://dx.doi.org/10.3354/meps07887

[130] Marubini, F., Gimona, A., Evans, P.G.H., Wright, P.J. and Pierce, G.J. (2009) Habitat Preference and Interannual Variability in Occurrence of the Harbour Porpoise Phocoena phocoena off Northwest Scotland. Marine Ecology Progress Series, 381, 297-310. http://dx.doi.org/10.3354/meps07893

[131] Smith, B.D., Braulik, G., Strindberg, S., Mansur, R., Diyan, M.A.A. and Ahmed, B. (2009) Habitat Selection of Freshwater-Dependent Cetaceans and the Potential Effects of Declining Freshwater Flows and Sea-Level Rise in Waterways of the Sundarbans Mangrove Forest, Bangladesh. Aquatic Conservation: Marine and Freshwater Ecosystems, 19, 209-225. http://dx.doi.org/10.1002/aqc.987

[132] Becker, E.A., Forney, K.A., Ferguson, K.A., Foley, D.G., Smith, R.C., Barlow, J. and Redfern, J.V. (2010) Comparing California Current Cetacean-Habitat Models Developed Using in Situ and Remotely Sensed Sea Surface Temperature 
Data. Marine Ecology Progress Series, 413, 163-183. http://dx.doi.org/10.3354/meps08696

[133] Cotté, C., Guinet, C., Taupier-Letage, I. and Petiau, E. (2010) Habitat Use and Abundance of Striped Dolphins in the Western Mediterranean Sea Prior to the Morbillivirus Epizootic Resurgence. Endangered Species Research, 12, 203214. http://dx.doi.org/10.3354/esr00301

[134] Embling, C.B., Gillibrand, P.A., Gordon, J., Shrimpton, J., Stevick, P.T. and Hammond, P.S. (2010) Using Habitat Models to Identify Suitable Sites for Marine Protected Areas for Harbour Porpoises (Phocoena phocoena). Biological Conservation, 143, 267-279. http://dx.doi.org/10.1016/j.biocon.2009.09.005

[135] Garraffo, G.V., Dans, S.L., Crespo, E.A., Degrati, M., Giudici, P. and Gagliardini, D.A. (2010) Dusky Dolphin: Modeling Habitat Selection. Journal of Mammalogy, 91, 54-65. http://dx.doi.org/10.1644/09-MAMM-A-105R1.1.

[136] Viddi, F.A., Hucke-Gaete, R., Torres-Florez, J.P. and Riberio, S. (2010) Spatial and Seasonal Variability in Cetacean Distribution in the Fjords of Northern Patagonua, Chile. ICES Journal of Marine Science, 67, 959-970. http://dx.doi.org/10.1093/icesjms/fsp288

[137] Corkeron, P.J., Minton, G., Collins, T., Findlay, K., Willson, A. and Baldwin, R. (2011) Spatial Models of Sparse Data to Inform Cetacean Conservation Planning: An Example from Oman. Endangered Species Research, 15, 39-52. http://dx.doi.org/10.3354/esr00367

[138] Garaffo, G.V., Dans, S.L., Pedraza, S.N., Degrati, M., Schiavini, A., Gonzalez, R. and Crespo, E.A. (2011) Modeling Habitat Use for Dusky Dolphin and Commerson's Dolphin in Patagonia. Marine Ecology Progress Series, 421, 217227. http://dx.doi.org/10.3354/meps08912

[139] Pirotta, E., Matthiopoulos, J., MacKenzie, M., Scott-Hayward, L. and Rendell, L. (2011) Modelling Sperm Whale Habitat Preference: A Novel Approach Combining Transect and Follow Data. Marine Ecology Progress Series, 436, 257272. http://dx.doi.org/10.3354/meps09236

[140] Ainley, D.G., Jongsomjit, D., Ballard, G., Thiele, D., Fraser, W.R. and Tynan, C.T. (2012) Modeling the Relationship of Antarctic Minke Whales to Major Ocean Boundaries. Polar Biology, 35, 281-290. http://dx.doi.org/10.1007/s00300-011-1075-1

[141] Anderwald, P., Evans, P.G.H., Dyer, R., Dale, A., Wright, P.J. and Hoelzel, A.R. (2012) Spatial Scale and Environmental Determinants in Minke Whale Habitat Use and Foraging. Marine Ecology Progress Series, 450, 259-274. http://dx.doi.org/10.3354/meps09573

[142] Azzelino, A., Panigada, S., Lanfredi, C., Zanardelli, M., Airoldi, S. and Notobartolo di Sciara, G. (2012) Predictive Habitat Models for Managing Marine Areas: Spatial and Temporal Distribution of Marine Mammals within the Pelagos Sanctuary (Northwestern Mediterranean Sea). Ocean and Coastal Management, 67, 63-74. http://dx.doi.org/10.1016/j.ocecoaman.2012.05.024

[143] Becker, E.A., Foley, D.G., Forney, K.A., Barlow, J., Redfern, J.V. and Gentemann, C.L. (2012) Forecasting Cetacean Abundance Patterns to Enhance Management Decisions. Endangered Species Research, 16, 97-112. http://dx.doi.org/10.3354/esr00390

[144] Best, B.D., Halpin, P.N., Read, A.J., Fujioka, E., Good, C.P., LaBrecque, E.A., Schick, R.S., Roberts, J.J., Hazen, L.J., Qian, S.S., Palka, D.L., Garrison, L.P. and McLellan, W.A. (2012) Online Cetacean Habitat Modelling System for the US East Coast and Gulf of Mexico. Endangered Species Research, 18, 1-15. http://dx.doi.org/10.3354/esr00430

[145] Dalla Rosa, L., Ford, J.K.B. and Trites, A.W. (2012) Distribution and Relative Abundance of Humpback Whales in Relation to Environmental Variables in Coastal British Columbia and Adjacent Waters. Continental Shelf Research, 36, 89-104. http://dx.doi.org/10.1016/j.csr.2012.01.017

[146] Doniol-Valcroze, T., Lesage, V., Giard, J. and Michaud, R. (2012) Challenges in Marine Mammal Habitat Modelling: Evidence of Multiple Foraging Habitats from the Identification of Feeding Events in Blue Whales. Endangered Species Research, 17, 255-268. http://dx.doi.org/10.3354/esr00427

[147] Forney, K.A., Ferguson, M.C., Becker, E.A., Fiedler, P.C., Redfern, J.V., Barlow, J., Vilchis, I.L. and Balance, L.T. (2012) Habitat-Based Spatial Models of Cetacean Density in the Eastern Pacific Ocean. Endangered Species Research, 16, 113-133. http://dx.doi.org/10.3354/esr00393

[148] Thorne, L.H., Johnston, D.W., Urban, D.L., Tyne, J., Bejder, L., Baird, R.W., Yin, S., Rickards, S.H., Deakos, M.H., Mobley Jr., J.R., Pack, A.A. and Chapla Hill, M. (2012) Predictive Modeling of Spinner Dolphin (Stenella longirostris) Resting Habitat in the Main Hawaiian Islands. PloS ONE, 7, e43167. http://dx.doi.org/10.1371/journal.pone.0043167

[149] Booth, C.G., Embling, C., Gordon, J., Calderan, S.V. and Hammond, P.S. (2013) Habitat Preferences and Distribution of the Harbour Porpoise Phocoena phocoena West of Scotland. Marine Ecology Progress Series, 478, 273-285. http://dx.doi.org/10.3354/meps10239

[150] MacLeod, C.D., Santos, M.B., Burns, F., Brownlow, A. and Pierce, G.J. (2013) Can Habitat Modelling for the Octopus Eledone cirrhosa Help Identify Key Areas for Risso’s Dolphin in Scottish Waters? Hydrobiologia, 725, 125-136. http://dx.doi.org/10.1007/s10750-013-1555-0 
[151] Torres, L.G., Smith, T.D., Sutton, P., MacDiarmid, A., Bannister, J. and Miyashita, T. (2013) From Exploitation to Conservation: Habitat Models Using Whaling Data Predict Distribution Patterns and Threat Exposure of an Endangered Whale. Diversity and Distributions, 19, 1138-1152. http://dx.doi.org/10.1111/ddi.12069 